Groups Geom. Dyn. 9 (2015), 737-782

DOI $10.4171 /$ GGD/326
Groups, Geometry, and Dynamics

(C) European Mathematical Society

\title{
On the character variety of the four-holed sphere
}

\author{
Sara Maloni, ${ }^{1}$ Frédéric Palesi, ${ }^{2}$ and Ser Peow Tan ${ }^{3}$
}

\begin{abstract}
We study the (relative) SL $(2, \mathbb{C})$ character varieties of the four-holed sphere and the action of the mapping class group on them. We describe a domain of discontinuity for this action, and, in the case of real characters, show that this domain of discontinuity may be non-empty on the components where the relative Euler class is non-maximal.
\end{abstract}

Mathematics Subject Classification (2010). Primary: 57M50; Secondary: 20E05, 37A15.

Keywords. Four-holed sphere, character variety, mapping class group, Bowditch set, domain of discontinuity, Markoff maps.

\section{Contents}

1 Introduction . . . . . . . . . . . . . . . . . . . . . . . . . . .

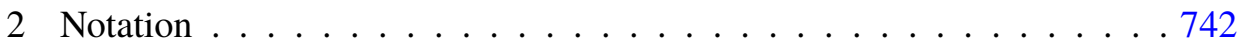

3 Estimates on Markoff maps . . . . . . . . . . . . . . . . . 749

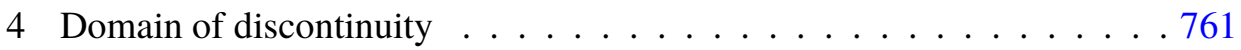

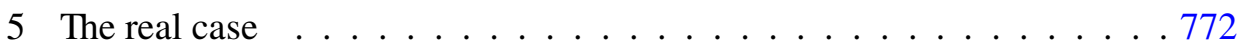

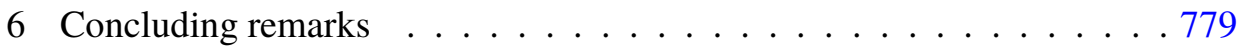

References...................... . . . . . . . . . . . . . . . . . . . .

${ }^{1}$ The first author was partially supported by the European Research Council under the European Community's seventh Framework Programme (FP7/2007-2013)/ERC grant agreement n FP7-246918.

2 The second author was partially supported by the ANR 2011 BS 0102001 ModGroup.

${ }^{3}$ The third author was partially supported by the National University of Singapore academic research grant R-146-000-156-112. 


\section{Introduction}

In his $\mathrm{Ph} . \mathrm{D}$. thesis [19], McShane established the following remarkable identity for lengths of simple closed geodesics on a once-punctured torus $S_{1,1}$ with a complete, finite area hyperbolic structure:

$$
\sum_{\gamma} \frac{1}{1+\exp (l(\gamma))}=\frac{1}{2}
$$

where $\gamma$ varies over all simple closed geodesics on $S_{1,1}$, and $l(\gamma)$ is the hyperbolic length of $\gamma$ under the given hyperbolic structure on $S_{1,1}$. This result was later generalized to (general) hyperbolic surfaces with cusps by McShane himself [20], to hyperbolic surfaces with cusps and/or geodesic boundary components by Mirzakhani [23], and to hyperbolic surfaces with cusps, geodesic boundary and/or conical singularities, as well as to classical Schottky groups by Tan, Wong and Zhang in [27], [29].

On the other hand, Bowditch in [4] gave an alternative proof of (1) via Markoff maps, and extended it in [6] to type-preserving representations of the once-punctured torus group into $\mathrm{SL}(2, \mathbb{C})$ satisfying certain conditions which we call here the BQ-conditions (Bowditch's Q-conditions). He also obtained in [5] a variation of (1) which applies to hyperbolic once-punctured torus bundles. Subsequently, Sakuma [25], Akiyoshi, Miyachi and Sakuma [1], [2] and recently Sakuma and Lee [17] refined Bowditch's results and generalized them to those which apply to hyperbolic punctured surface bundles. In [28] Tan, Wong and Zhang also further extended Bowditch's results to representations of the once-punctured torus group into $\mathrm{SL}(2, \mathbb{C})$ which are not type-preserving, that is, where the commutator is not parabolic, and also to representations which are fixed by an Anosov element of the mapping class group and which satisfy a relative version of the Bowditch's Q-conditions. They also showed that the BQ-conditions defined an open subset of the character variety on which the mapping class group of the punctured torus acted properly discontinuously.

In a different direction, Labourie and McShane in [16] showed that the identity above has a natural formulation in terms of (generalised) cross ratios, and then, using this formulation, studied identities arising from the cross ratios constructed by Labourie for representations from fundamental groups of surfaces to $\operatorname{PSL}(n, \mathbb{R})$.

The above papers provided much of the motivation for this paper, in particular, the identities obtained were in many cases valid for the moduli spaces of hyperbolic structures, so invariant under the action of the mapping class group, and in the case of cone structures, they could be interpreted as identities valid for certain subsets of the character variety which were invariant under the action of the 
mapping class group, even though the representations in the subset may be nondiscrete or non-faithful. This leads naturally to the question of whether there were interesting subsets of the character varieties on which the mapping class group acts properly discontinuously, but which consists of more than just discrete, faithful representations, as explored in the punctured torus case in [28].

In this paper we will consider representations of the free group on three generators $F_{3}=\langle\alpha, \beta, \gamma, \delta: \alpha \beta \gamma \delta=I\rangle$ into $\operatorname{SL}(2, \mathbb{C})$. We adopt the viewpoint that $F_{3}$ is the fundamental group of the four-holed sphere $S$, with $\alpha, \beta, \gamma, \delta$ identified with $\partial S$, and study the natural action of $\mathcal{M C G}(S)$, the mapping class group of $S$ on the character variety

$$
X:=\operatorname{Hom}\left(F_{3}, \operatorname{SL}(2, \mathbb{C})\right) / / \operatorname{SL}(2, \mathbb{C}),
$$

where we take the quotient in the sense of geometric invariant theory. If $\theta \in$ $\mathcal{M C G}(S)$ and $[\rho] \in \mathcal{X}$, this action is given by

$$
\theta([\rho])=\left[\rho \circ\left(\theta_{*}\right)^{-1}\right],
$$

where $\theta_{*}: \pi_{1}(S) \rightarrow \pi_{1}(S)$ is the map associated to $\theta$ in homotopy. We are interested in the dynamics of this action, in particular, on the relative character varieties $x_{(a, b, c, d)}$, which is the set of representations for which the traces of the boundary curves are fixed.

We describe the following result, see Theorems 4.19 and 4.20.

Theorem A. There exists a domain of discontinuity for the action of $\mathcal{M C G}(S)$ on $X_{(a, b, c, d)}$, that is, an open $\mathcal{M C G}(S)$-invariant subset $D \subset X_{(a, b, c, d)}$ on which $\mathcal{M C G}(S)$ acts properly discontinuously.

Remark 1.1. As already observed by several other authors in related situations (see Goldman [12], Tan, Wong, and Zhang [28], and Minsky [21]), our domain of discontinuity contains the interior of the discrete and faithful characters, but also characters which may not be discrete or faithful. For example, when the boundary traces are in $(-2,2)$ we can produce representations that are non-discrete, but are nevertheless in the domain of discontinuity.

A much deeper question is about the case of non-elliptic boundary values. In that situation, we don't know the complete answer, and the question is certainly not easy. For example, in the case $X_{-2}\left(\Sigma_{1,1}\right)$ Bowditch conjectured that this set coincides with the quasifuchsian representations, see [6].

This set is described by two conditions, much in the spirit of [6] and [28], and is given as follows. If $\mathcal{S}$ denotes the set of free homotopy classes of essential, 
non-peripheral simple closed curves on $S$, then the conditions for $[\rho]$ to be in $D$ are

(i) $\operatorname{tr} \rho(\gamma) \notin[-2,2]$ for all $\gamma \in \mathcal{S}$;

(ii) $|\operatorname{tr} \rho(\gamma)|<K$ for only finitely many $\gamma \in \mathcal{S}$, where $K>0$ is a fixed constant that depends only on $a, b, c, d$.

Furthermore, the set of $\gamma$ satisfying condition (ii) above satisfy a quasi-convexity property, equivalently, is connected when represented as the subset of the complementary regions of a properly embedded binary tree (see Theorem 3.1). This property is particularly important when writing a computer program to draw slices of the domain of discontinuity.

Of particular interest is the set of real characters, which consists of representations in SL(2, R) or SU(2). In the latter case, Goldman [11] proved ergodicity of the mapping class group action for all orientable hyperbolizable surfaces, with respect to the invariant measure induced by the natural symplectic structure on the moduli space. (This was generalized by the second author in the non-orientable case in [24]). On the other hand, in the $\operatorname{SL}(2, \mathbb{R})$ case the dynamics is much richer and less understood. For example, when $S_{g}$ is a closed surface of genus $g \geq 2$, Goldman conjectured that the action of $\mathcal{M C G}\left(S_{g}\right)$ on the components of $\mathcal{X}\left(S_{g}\right)$ with non-maximal Euler class is ergodic. An approach towards a proof of this would be to use a cut-and-paste argument involving pieces homeomorphic to one-holed tori and four-holed spheres. While the case of the one-holed torus was completely described by Goldman in [12], we obtain partial results in the four-holed sphere case here. In fact, an important corollary of our analysis is the following (see Theorem 5.3):

Theorem B. In the real case, for all boundary datas, except a dimension one subset, there is a non-empty open domain of discontinuity for the action of $\mathcal{M C G}(S)$ on the relative $\mathrm{SL}(2, \mathbb{R})$-character variety.

This implies that there are representations in these components for which all essential simple closed curves on $S$ have hyperbolic representatives, even though these representations may not be discrete and faithful. There are also some surprises here, in particular, certain slices of the real character variety satisfying some general condition always have non-empty intersection with the domain of discontinuity. 
The mapping class group $\mathcal{M} \mathcal{C G}(S)$ consisting of equivalence classes of diffeomorphisms of $S$ fixing the boundary is isomorphic to the index two normal subgroup of the triangle group $\mathbb{Z}_{2} * \mathbb{Z}_{2} * \mathbb{Z}_{2}$ generated by reflections on an ideal triangle and it acts on the character variety as given in the earlier part of the introduction. For a representation $\rho: F_{3} \rightarrow \operatorname{SL}(2, \mathbb{C})$, we can look at its character

$$
\begin{aligned}
\chi_{\rho}: F_{3} & \longrightarrow \mathbb{C}, \\
W & \longmapsto \operatorname{tr}(\rho(W)) .
\end{aligned}
$$

The character variety is exactly the set of characters, by results of [18], see for example [12], and each character is in turn determined entirely by its value on the seven elements as follows, satisfying equation (2):

$$
\begin{gathered}
\chi_{\rho}(\alpha):=a, \quad \chi_{\rho}(\beta):=b, \quad \chi_{\rho}(\gamma):=c, \quad \chi_{\rho}(\delta):=d, \\
\chi_{\rho}(\alpha \beta):=x, \quad \chi_{\rho}(\beta \gamma):=y, \quad \chi_{\rho}(\gamma \alpha):=z .
\end{gathered}
$$

Hence, we identify $X$ with the variety $\mathcal{V}$ consisting of points $(a, b, c, d, x, y, z) \in$ $\mathbb{C}^{7}$ satisfying the equation

$$
x^{2}+y^{2}+z^{2}+x y z=p x+q y+r z+s,
$$

where

$$
\begin{aligned}
& p=a b+c d, \\
& q=b c+a d, \\
& r=a c+b d, \\
& s=4-a^{2}-b^{2}-c^{2}-d^{2}-a b c d .
\end{aligned}
$$

In particular, this identifies the character variety with the branched double cover of $\mathbb{C}^{6}$, which is homotopic to a 6-sphere, see [7].

The $\mathcal{M C G}(S)$ action extends to an action of $\mathbb{Z}_{2} * \mathbb{Z}_{2} * \mathbb{Z}_{2}$ on $\mathcal{V}$, which is generated by

$$
\theta_{1}, \theta_{2}, \theta_{3}: \mathcal{V} \rightarrow \mathcal{V}
$$

where

$$
\begin{aligned}
& \theta_{1}(a, b, c, d, x, y, z)=(a, b, c, d, p-y z-x, y, z), \\
& \theta_{2}(a, b, c, d, x, y, z)=(a, b, c, d, x, q-x z-y, z), \\
& \theta_{3}(a, b, c, d, x, y, z)=(a, b, c, d, x, y, r-x y-z) .
\end{aligned}
$$


Elements of the mapping class group correspond to words of even length in $\theta_{1}, \theta_{2}$ and $\theta_{3}$, in particular, $\theta_{2} \theta_{3}, \theta_{3} \theta_{1}$ and $\theta_{1} \theta_{2}$ correspond to Dehn twists about essential simple closed curves on $S$ and generate the $\mathcal{M C G}(S)$ action on $\mathcal{V}$.

It is somewhat remarkable that many of the results of [6] and [28] generalize to the problem we study here, although the analysis is necessarily more technical and complicated, but in some sense, also more interesting. There are however some results which do not generalize, see Example 3.11. Finally, we note that recent work of Hu, Tan and Zhang [14] on Coxeter group actions on varieties defined by the Markoff-Hurwitz equation indicate that in fact, there should be a deeper underlying theory for analyzing the domains of discontinuity for group actions of this type.

The rest of this paper is organized as follows. In $\S 2$ we set the notation and give some basic definitions. In $\S 3$ we prove the generalizations of the basic lemmas (in terms of Markoff maps) required to analyse and understand the orbit of a character under the action of the mapping class group. In particular, generalizations of the "fork" lemma (Lemma 3.3) and the quasi-convexity result (Theorem 3.1) from [6] and [28], as well as an analysis of the values taken by the neighbors around a region are covered. In $\S 4$ we give a proof of our main theorem (Theorem A) which describes the domain of discontinuity for the action in terms of the BQ-conditions. In $\S 5$, we consider the real case and show that domains of discontinuity can occur in the components of the $\operatorname{PSL}(2, \mathbb{R})$ relative character variety which do not have maximal relative Euler class (Theorem B). Finally, in $\S 6$ we give some concluding remarks.

Acknowledgements. This project was initiated when the authors were participating in the trimester program on "Geometry and Analysis of surface group representations" at the Institut Henri Poincaré (Jan-Mar 2012). The authors are grateful to the organizers of the program for the invitations to participate in the program, and to the IHP and its staff for their hospitality and generous support. In particular, we would like to thank Bill Goldman and Richard Canary for helpful conversations, and the anonymous referee for the very careful reading of the manuscript and the many helpful comments and suggestions.

\section{Notation}

In this section we set the notation which we will use in the paper and give some important definitions. Since many of the results included in this article are influenced 
by, and are generalisations of the results of Tan, Wong and Zhang's article [28], we will try to follow closely the notation and structure of that paper, so that the interested reader will find it easier to compare our results with theirs. We should also note that their paper was a generalisation of Bowditch's results [6].

2.1. The four-holed sphere group. Let $S=S_{0,4}$ be a (topological) four-holed sphere, that is, a sphere with four (disjoint) open disks removed, and let $\Gamma$ be its fundamental group. The group $\Gamma$ admits the following presentation

$$
\Gamma=\langle\alpha, \beta, \gamma, \delta \mid \alpha \beta \gamma \delta\rangle
$$

where $\alpha, \beta, \gamma$ and $\delta$ correspond to homotopy classes of the four boundary components, one for each removed disk. Note that $\Gamma$ is isomorphic to the free group on three generators $\mathbb{Z} * \mathbb{Z} * \mathbb{Z}=\langle\alpha, \beta, \gamma\rangle$ since $\delta=(\alpha \beta \gamma)^{-1}$.

We define an equivalence relation $\sim$ on $\Gamma$ by $g \sim h$ if and only if $g$ is conjugate to $h$ or $h^{-1}$. Note that $\Gamma / \sim$ can be identified with the set of free homotopy classes of unoriented closed curves on $S$.

2.2. Simple closed curves on the sphere. Let $\mathcal{S}=\mathcal{S}(S)$ be the set of free homotopy classes of essential (that is, non-trivial and non-peripheral) simple closed curves on $S$ and let $\widehat{\Omega} \subset \Gamma / \sim$ be the subset corresponding to $\mathcal{S}$. Note that $\widehat{\Omega}$ can be identified with $\widehat{\mathbb{Q}}=\mathbb{Q} \cup\{\infty\}$ by considering the 'slope' of $[g] \in \hat{\Omega}$, see, among others, Proposition 2.1 of Keen and Series [15]. For example, we can identify $\alpha \beta$ with $0, \beta \gamma$ with $\infty, \alpha \gamma$ with -1 , and so on.

We also observe that $\hat{\Omega}$ inherits a cyclic ordering from the cyclic ordering of $\widehat{\mathbb{Q}}$ induced from the standard embedding into $\widehat{\mathbb{R}}=\mathbb{R} \cup\{\infty\} \cong S^{1}$.

2.3. Relative character variety of $\Gamma$. The character variety $X=X(\Gamma, \operatorname{SL}(2, \mathbb{C}))$ is the space of equivalence classes of representations $\rho: \Gamma \rightarrow \operatorname{SL}(2, \mathbb{C})$, where the equivalence classes are obtained by taking the closure of the orbit under the conjugation action by $\operatorname{SL}(2, \mathbb{C})$.

A representation

$$
\rho: \Gamma \longrightarrow \operatorname{SL}(2, \mathbb{C})
$$

is said to be a $\boldsymbol{\tau}$-representation, or a $\boldsymbol{\tau}$-character, where $\boldsymbol{\tau}=(a, b, c, d) \in \mathbb{C}^{4}$, when for some fixed generators $\alpha, \beta, \gamma, \delta \in \Gamma$ corresponding to the boundary com- 
ponents of $S$,

$$
\begin{aligned}
& \operatorname{tr} \rho(\alpha)=a, \\
& \operatorname{tr} \rho(\beta)=b, \\
& \operatorname{tr} \rho(\gamma)=c, \\
& \operatorname{tr} \rho(\delta)=d .
\end{aligned}
$$

The space of equivalence classes of $\boldsymbol{\tau}$-representations is denoted by $X_{\boldsymbol{\tau}}$ and is called the $\boldsymbol{\tau}$-relative character variety. These representations correspond to representations of the four-holed sphere where we fix the conjugacy classes of the four boundary components in the space of closed orbits.

For $\rho \in X_{\boldsymbol{\tau}}$, we let

$$
\begin{aligned}
& x=\operatorname{tr}(\rho(\alpha \beta)), \\
& y=\operatorname{tr}(\rho(\beta \gamma)),
\end{aligned}
$$

and

$$
z=\operatorname{tr}(\rho(\alpha \gamma)) .
$$

A classical result on the character varieties (see (9) in Fricke and Klein [9], p. 298) states that $x_{\tau}$ is identified with the set

$$
\left\{(x, y, z) \in \mathbb{C}^{3}: x^{2}+y^{2}+z^{2}+x y z=p x+q y+r z+s\right\},
$$

where

$$
\begin{aligned}
& p=a b+c d, \\
& q=b c+a d, \\
& r=a c+b d, \\
& s=4-a^{2}-b^{2}-c^{2}-d^{2}-a b c d .
\end{aligned}
$$

The mapping class group of $S$,

$$
\mathcal{M C G}:=\pi_{0}(\operatorname{Homeo}(S))
$$

acts on $X_{\boldsymbol{\tau}}$, see [12]. For concreteness, we adopt the convention here that $\mathcal{M} C \mathcal{G}$ consists of orientation-preserving homeomorphisms fixing the boundary, there will be no essential difference to the ensuing discussion. The mapping class group 
is generated by Dehn twists along the simple closed curves corresponding to $\alpha \beta$, $\beta \gamma$ and $\alpha \gamma$. The action of each Dehn twist can be read easily on the trace coordinates of $X_{\boldsymbol{\tau}}$. For example the Dehn twist about the separating curve $\alpha \beta$ is the map $\mathbb{C}^{3} \rightarrow \mathbb{C}^{3}$ given by

$$
\left(\begin{array}{l}
x \\
y \\
z
\end{array}\right) \longmapsto\left(\begin{array}{c}
x \\
q-x(r-x y-z)-y \\
r-x y-z
\end{array}\right),
$$

where $p, q, r$ are defined as above. This corresponds to the action of $\theta_{2} \theta_{3}$ given in the Introduction, see also Remark 3.6.

2.4. The BQ-conditions. For a fixed $\boldsymbol{\tau}$, let $K=K(\boldsymbol{\tau})>0$ be a constant depending only on $\boldsymbol{\tau}$, that we will define later in Definition 3.9. A $\boldsymbol{\tau}$-representation $\rho: \Gamma \rightarrow \operatorname{SL}(2, \mathbb{C})$ is said to satisfy the $B Q$-conditions (Bowditch's Q-conditions) if

(BQ1) $\operatorname{tr} \rho(g) \notin[-2,2]$ for all $[g] \in \widehat{\Omega}$;

(BQ2) $|\operatorname{tr} \rho(g)| \leq K$ for only finitely many (possibly no) $[g] \in \widehat{\Omega}$.

We call such a representation $\rho$ a BQ-representation, or Bowditch representation, and the space of equivalence classes of such representations the Bowditch representation space, denoted by $\left(X_{\tau}\right)_{Q}$.

Note that $\operatorname{tr} \rho\left(g_{1}\right)=\operatorname{tr} \rho\left(g_{2}\right)$ if $\left[g_{1}\right]=\left[g_{2}\right]$ (since $g_{1}$ is conjugate to $g_{2}$ or its inverse by definition); so the conditions (BQ1) and (BQ2) make sense.

2.5. The binary tree $\Sigma$. Let $\Sigma$ be a countably infinite simplicial tree properly embedded in the plane all of whose vertices have degree 3. As an example, we can consider, as $\Sigma$, the binary tree dual to the Farey triangulation $\mathcal{F}$ of the hyperbolic plane $\mathbb{H}^{2}$ (also called an infinite trivalent tree). See [28] for the definition of $\mathcal{F}$.

2.6. Complementary regions. A complementary region of $\Sigma$ is the closure of a connected component of the complement.

We denote by

$$
\Omega=\Omega(\Sigma)
$$

the set of complementary regions of $\Sigma$. Similarly, we use $V(\Sigma), E(\Sigma)$ for the set of vertices and edges of $\Sigma$ respectively. 


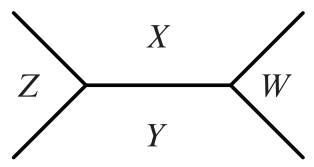

Figure 1. The edge $e \leftrightarrow(X, Y ; Z, W)$.

We use the letters $X, Y, Z, W, \ldots$ to denote the elements of $\Omega$. For $e \in E(\Sigma)$, we also use the notation $e \leftrightarrow(X, Y ; Z, W)$ to indicate that $e=X \cap Y$ and $e \cap Z$ and $e \cap W$ are the endpoints of $e$; see Figure 1.

2.7. A tri-coloring of the tree. We choose a coloring of the regions and edges, namely a map

$$
\mathcal{C} \Omega(\Sigma) \cup E(\Sigma) \longrightarrow\{1,2,3\}
$$

such that for any edge $e \leftrightarrow(X, Y ; Z, W)$ we have $\mathcal{C}(e)=\mathcal{C}(Z)=\mathcal{C}(W)$ and such that $\mathrm{C}(e), \mathrm{C}(X), \mathrm{C}(Y)$ are all different. The coloring is completely determined by a coloring of the three regions around any specific vertex, and hence is unique up to a permutation of the set $\{1,2,3\}$. We denote by $\Omega_{i}(\Sigma)$ the set of complementary regions with color $i$, and by $E_{i}(\Sigma)$ the set of edges with color $i$.

As a convention, in the following, when $X, Y, Z$ are complementary regions around a vertex, we will have $X \in \Omega_{1}(\Sigma), Y \in \Omega_{2}(\Sigma)$, and $Z \in \Omega_{3}(\Sigma)$.

2.8. $\boldsymbol{\mu}$-Markoff triples. For a complex quadruple $\boldsymbol{\mu}=(p, q, r, s) \in \mathbb{C}^{4}$, a $\mu$-Markoff triple is an ordered triple $(x, y, z)$ of complex numbers satisfying the $\mu$-Markoff equation:

$$
x^{2}+y^{2}+z^{2}+x y z=p x+q y+r z+s .
$$

Note that, if $(x, y, z)$ is a $\mu$-Markoff triple in the sense of Tan, Wong, and Zhang, then $(-x,-y,-z)$ is a $(0,0,0, \mu)$-Markoff triple in our sense.

It is easily verified that, if $(x, y, z)$ is a $\mu$-Markoff triple, so are the triples

$$
(x, y, r-x y-z), \quad(x, q-x z-y, z), \quad(p-y z-x, y, z) .
$$

It is important to note that permutations triples are not $\boldsymbol{\mu}$-Markoff triples, contrary to the situation with $\mu$-Markoff triples. 
2.9. Relation with $\tau$-representations. Let GT: $\mathbb{C}^{4} \longrightarrow \mathbb{C}^{4}$ be the map defined by

$$
\left[\begin{array}{l}
a \\
b \\
c \\
d
\end{array}\right] \longmapsto\left[\begin{array}{c}
a b+c d \\
a d+b c \\
a c+b d \\
4-a^{2}-b^{2}-c^{2}-d^{2}-a b c d
\end{array}\right]=\left[\begin{array}{l}
p \\
q \\
r \\
s
\end{array}\right] .
$$

This map is defined and studied by by Cantat and Loray in [8] and by Goldman and Toledo in [13], where they show, among many other results, that the map is onto and proper. (Note that Goldman and Toledo denote this map $\Phi$.)

Remark 2.1. Given $\tau \in \mathbb{C}^{4}$, a representation $\rho$ is in $X_{\tau}$ if and only if the triple $(\operatorname{tr}(\rho(\alpha \beta)), \operatorname{tr}(\rho(\beta \gamma)), \operatorname{tr}(\rho(\alpha \gamma)))$ is a $\boldsymbol{\mu}$-Markoff triple with $\boldsymbol{\mu}=\mathrm{GT}(\boldsymbol{\tau})$.

The elementary operations defined in (4) are intimately related with the action of the mapping class group on the character variety, as we will see later.

2.10. $\boldsymbol{\mu}$-Markoff map. A $\boldsymbol{\mu}$-Markoff map is a function

$$
\phi: \Omega \longrightarrow \mathbb{C}
$$

such that

(i) for every vertex $v \in V(\Sigma)$, the triple $(\phi(X), \phi(Y), \phi(Z))$ is a $\mu$-Markoff triple, where $X, Y, Z \in \Omega$ are the three regions meeting $v$ such that $X \in \Omega_{1}$, $Y \in \Omega_{2}$ and $Z \in \Omega_{3}$;

(ii) for any $i \in\{1,2,3\}$ and for every edge $e \in E_{i}(\Sigma)$ we have

- If $i=1$ and $e \leftrightarrow\left(Y, Z ; X, X^{\prime}\right)$, then

$$
x+x^{\prime}=p-y z,
$$

- If $i=2$ and $e \leftrightarrow\left(X, Z ; Y, Y^{\prime}\right)$, then

$$
y+y^{\prime}=q-x z
$$

- If $i=3$ and $e \leftrightarrow\left(X, Y ; Z, Z^{\prime}\right)$, then

$$
z+z^{\prime}=r-x y,
$$

where $x=\phi(X), y=\phi(Y), z=\phi(Z), x^{\prime}=\phi\left(X^{\prime}\right), y^{\prime}=\phi\left(Y^{\prime}\right)$ and $z^{\prime}=\phi\left(Z^{\prime}\right)$ 
Note that if condition (i) is satisfied at one vertex, then condition (ii) guarantees that it is satisfied at every vertex. This is related to the fact that the edge relations in fact arise from mapping class group actions of the four-holed sphere which preserve the boundary traces, and hence the relative character variety.

We shall use $\boldsymbol{\Phi}_{\boldsymbol{\mu}}$ to denote the set of all $\boldsymbol{\mu}$-Markoff maps and lower case letters to denote the $\phi$ values of the regions. For example, we have $\phi(X)=x, \phi(Y)=$ $y, \phi(Z)=z$.

Remark 2.2. There exists a bijective correspondence between $\boldsymbol{\mu}$-Markoff maps and $\boldsymbol{\mu}$-Markoff triples. Hence, using Remark 2.1, there exists a bijective correspondence between the set $\boldsymbol{\Phi}_{\mu}$ of $\boldsymbol{\mu}$-Markoff maps and the $\boldsymbol{\tau}$-relative character variety $X_{\tau}$, where $\mu=\mathrm{GT}(\boldsymbol{\tau})$.

In fact, as in the case of Markoff maps and $\mu$-Markoff maps, if the edge relations (5), (6) and (7) are satisfied along all edges, then it suffices that the vertex relation (3) is satisfied at a single vertex. So one may establish a bijective correspondence between $\boldsymbol{\mu}$-Markoff maps and $\boldsymbol{\mu}$-Markoff triples, by fixing three regions $X, Y, Z$ which meet at some vertex $v_{0}$. This process may be inverted by constructing a tree of $\boldsymbol{\mu}$-Markoff triples as Bowditch did in [6] for Markoff triples and as Tan, Wong and Zhang did in [28] for the $\mu$-Markoff triples: given a triple $(x, y, z)$, set $\phi(X)=x, \phi(Y)=y, \phi(Z)=z$, and extend over $\Omega$ as dictated by the edge relations. In this way one obtains an identification of $\boldsymbol{\Phi}_{\boldsymbol{\mu}}$ with the algebraic variety in $\mathbb{C}^{3}$ given by the $\boldsymbol{\mu}$-Markoff equation. In particular, $\boldsymbol{\Phi}_{\boldsymbol{\mu}}$ gets an induced topology as a subset of $\mathbb{C}^{3}$.

2.11. The subsets $\boldsymbol{\Omega}_{\boldsymbol{\phi}}(\boldsymbol{k}) \subset \boldsymbol{\Omega}$. Given $\phi \in \boldsymbol{\Phi}_{\boldsymbol{\mu}}$ and $k \geq 0$, the set $\Omega_{\phi}(k) \subseteq \Omega$ is defined by

$$
\Omega_{\phi}(k)=\{X \in \Omega:|\phi(X)| \leq k\} .
$$

These sets will be crucial in the proof of our main results.

We can now state the BQ-conditions in terms of Markoff maps.

Definition 2.3. For a fixed $\mu$, let $L=L(\mu)>0$ be a constant depending only on $\boldsymbol{\mu}$, that we will define later in Definition 3.9. A $\boldsymbol{\mu}$-Markoff map $\phi \in \boldsymbol{\Phi}_{\boldsymbol{\mu}}$ is said to satisfy the $B Q$-conditions if

(BQ1) $\phi^{-1}([-2,2])=\emptyset$;

(BQ2) $\Omega_{\phi}(L)$ is finite. 
We denote by $\left(\boldsymbol{\Phi}_{\boldsymbol{\mu}}\right)_{Q}$ the set of all $\boldsymbol{\mu}$-Markoff maps which satisfy the BQ-conditions, and call the set of all such maps the Bowditch $\mu$-Markoff maps.

We will see that it is sufficient to give a large enough constant $L$ to define the BQ-conditions. Indeed, taking a bigger constant $L^{\prime}>L(\boldsymbol{\mu})$ in the definition will give rise to the same subset of Markoff maps.

\section{Estimates on Markoff maps}

The main aim of this section is to prove the following result, which is a generalisation of Theorem 3.1 of Tan, Wong, and Zhang [28].

Theorem 3.1 (quasi-convexity). Let $\mu \in \mathbb{C}^{4}$.

(1) There exists a constant $l=l(\boldsymbol{\mu})>0$ such that for all $\phi \in \boldsymbol{\Phi}_{\boldsymbol{\mu}}$, we have that $\Omega_{\phi}(l)$ is non-empty.

(2) There exists a constant $\alpha=\alpha(\boldsymbol{\mu}) \geq 0$ such that for all $\phi \in \boldsymbol{\Phi}_{\boldsymbol{\mu}}$ and all $k \geq 2+\alpha$, the set $\Omega_{\phi}(k)$ is connected.

For doing that, we need to do lots of estimates. In order to shorten a bit the formulae which will appear, we introduce the following notation.

Notation 3.2. Given a $\mu \in \mathbb{C}^{4}$, let

$$
\alpha=\alpha(\mu)=\frac{\max \{|p|,|q|,|r|\}}{2} .
$$

For the rest of this section, let us fix $\mu \in \mathbb{C}^{4}$ and a $\mu$-Markoff map $\phi$.

3.1. Arrows assigned by a $\boldsymbol{\mu}$-Markoff map. As Bowditch [6] and Tan, Wong and Zhang [28] did, we may use $\phi \in \boldsymbol{\Phi}_{\boldsymbol{\mu}}$ to assign to each undirected edge $e$ a particular directed edge vect ${ }_{\phi}(e)$ with underlying edge $e$. Suppose $e \leftrightarrow(X, Y ; Z, W)$. If $|z|>|w|$, then the arrow on $e$ points towards $W$; in other words, $\operatorname{vect}_{\phi}(e)=$ $(X, Y ; Z \rightarrow W)$. If $|z|<|w|$, we put an arrow on $e$ pointing towards $Z$, that is,

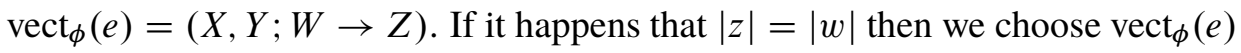
arbitrarily, the choice does not affect the arguments in the latter part of this paper. Let $\vec{E}(\Sigma)$ be the set of oriented edges.

A vertex with all three arrows pointing towards it is called a sink, one where two arrows point towards it and one away is called a merge, and vertex with two (respectively three) arrows pointing away from it is called a fork (respectively source). 
Lemma 3.3 (fork lemma). Suppose $(X, Y, Z) \in \Omega_{1} \times \Omega_{2} \times \Omega_{3}$ meet at a vertex $v \in V(\Sigma)$, and the arrows on the edges $X \cap Y$ and $X \cap Z$ both point away from $v$. Then at least one of the following is true:

- $|x| \leq 2+\frac{|q|+|r|}{4}$;

- $|y|<2$;

- $|z|<2$.

We have similar results if the edges $Y \cap Z$ and $Y \cap X$ (or $Z \cap X$ and $Z \cap Y$ ) both point away from $v$.

Proof. Let $e_{2}$ and $e_{3}$ be the arrows pointing away from $v$, as shown in Figure 2.

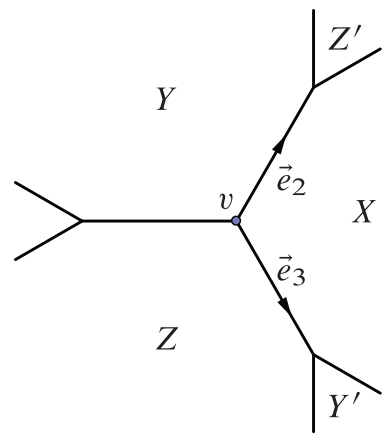

Figure 2. A fork.

Let $Y^{\prime}$ and $Z^{\prime}$ be the regions such that

$$
e_{2}=\left(X, Z ; Y \longrightarrow Y^{\prime}\right)
$$

and

$$
e_{3}=\left(X, Y ; Z \longrightarrow Z^{\prime}\right) \text {. }
$$

The edge relation gives

$$
\begin{aligned}
|x z| & =\left|y+y^{\prime}-q\right| \\
& \leq|y|+\left|y^{\prime}\right|+|q| \\
& \leq 2|y|+|q| .
\end{aligned}
$$

Similarly

$$
|x y| \leq 2|z|+|r| .
$$


Adding both inequalities, one obtains

$$
|x|(|y|+|z|) \leq 2(|y|+|z|)+(|q|+|r|) .
$$

If $|y| \geq 2$ and $|z| \geq 2$, then we have

$$
|x| \leq 2+\frac{|q|+|r|}{|y|+|z|} \leq 2+\frac{|q|+|r|}{4} .
$$

A weaker statement will be sufficient for most of the paper.

Corollary 3.4. Suppose $(X, Y, Z) \in \Omega_{1} \times \Omega_{2} \times \Omega_{3}$ meet at a vertex $v \in V(\Sigma)$, and two arrows point away from $v$, that is, $v$ is a fork or a source. Then

$$
\min \{|x|,|y|,|z|\} \leq 2+\alpha .
$$

Lemma 3.5. There is a constant $m(\boldsymbol{\mu}) \in \mathbb{R}_{>0}$ such that if three regions $X, Y, Z$ meet at a sink, then

$$
\min \{|x|,|y|,|z|\} \leq m(\boldsymbol{\mu}) .
$$

Proof. We show that, if $|x|,|y|,|z|$ are all sufficiently large, then the vertex $v$ cannot be a sink. We may assume $x, y, z \neq 0$ and

$$
\max \{|p|,|q|,|r|\} \leq|x| \leq|y| \leq|z| .
$$

We can rewrite (3) as

$$
\frac{z}{x y}+\frac{y}{x z}+\frac{x}{y z}+1=\frac{p}{y z}+\frac{q}{x z}+\frac{r}{x y}+\frac{s}{x y z} .
$$

There exists $K>0$ such that if $K<|x| \leq|y| \leq|z|$, then

$$
\left|\frac{y}{x z}\right|, \quad\left|\frac{x}{y z}\right|, \quad\left|\frac{p}{y z}\right|, \quad\left|\frac{q}{x z}\right|, \quad\left|\frac{r}{x y}\right|, \quad\left|\frac{s}{x y z}\right|
$$

are all smaller than $\frac{1}{12}$ where the bounds for $\left|\frac{y}{x z}\right|$ and $\left|\frac{x}{y z}\right|$ follows from the fact that $|x| \leq|y| \leq|z|$ and $|x|,|y|,|z|>K$ with $K$ large (say $K>12$ ). It follows that

$$
\left|\frac{z}{x y}+1\right|<\frac{1}{2} \text { and so } \quad\left|\frac{z}{x y}\right|>\frac{1}{2} .
$$

On the other hand, we have

$$
\left|\frac{z}{x y}+1-\frac{r}{x y}\right|<\frac{1}{2} .
$$

So we infer that $|z|>|z+x y-r|$. Hence the arrow on the edge $X \cap Y$ is directed away from $v$ which proves that $v$ is not a sink. 
3.2. Neighbors around a region. For each $X \in \Omega_{1}$, its boundary $\partial X$ is a bi-infinite path consisting of a sequence of edges of the form $X \cap Y_{n}$ alternating with $X \cap Z_{n}$, where $\left(Y_{n}\right)_{n \in \mathbb{Z}}$ and $\left(Z_{n}\right)_{n \in \mathbb{Z}}$ are bi-infinite sequences of complementary regions in $\Omega_{2}$ and $\Omega_{3}$. The edge relations (6) and (7) are such that

$$
\begin{aligned}
y_{n+1} & =q-x z_{n}-y_{n} ; \\
z_{n+1} & =r-x y_{n+1}-z_{n} \\
& =r-x q+\left(x^{2}-1\right) z_{n}+x y_{n} .
\end{aligned}
$$

Remark 3.6. The map $\left(x, y_{n}, z_{n}\right) \rightarrow\left(x, y_{n+1}, z_{n+1}\right)$ is exactly the map defined by the Dehn twist along the curve $\alpha \beta$ defined in the introduction.

We can reformulate these equations in terms of matrices:

$$
\left(\begin{array}{l}
y_{n+1} \\
z_{n+1}
\end{array}\right)=\left(\begin{array}{cc}
-1 & -x \\
x & x^{2}-1
\end{array}\right) \cdot\left(\begin{array}{l}
y_{n} \\
z_{n}
\end{array}\right)+\left(\begin{array}{c}
q \\
r-q x
\end{array}\right) .
$$

If $x \neq \pm 2$, this can be rewritten as

$$
\left(\begin{array}{l}
y_{n+1} \\
z_{n+1}
\end{array}\right)=\left(\begin{array}{l}
\mathfrak{y}(x) \\
\mathfrak{z}(x)
\end{array}\right)+\left(\begin{array}{cc}
-1 & -x \\
x & x^{2}-1
\end{array}\right) \cdot\left[\left(\begin{array}{l}
y_{n} \\
z_{n}
\end{array}\right)-\left(\begin{array}{l}
\mathfrak{y}(x) \\
\mathfrak{z}(x)
\end{array}\right)\right],
$$

with

$$
\begin{aligned}
& \mathfrak{y}(x)=\frac{1}{4-x^{2}}(2 q-x r), \\
& \mathfrak{z}(x)=\frac{1}{4-x^{2}}(2 r-x q) .
\end{aligned}
$$

Note. $\mathfrak{y}(x)$ and $\mathfrak{z}(x)$ are the coordinates of the center of the conic in coordinates $(y, z)$ defined by the vertex relation.

The matrix

$$
M:=\left(\begin{array}{cc}
-1 & -x \\
x & x^{2}-1
\end{array}\right)
$$

has determinant one. Hence its eigenvalues $\lambda$ and $\lambda^{-1}$ are such that $\lambda+\lambda^{-1}=$ $\operatorname{tr} M=x^{2}-2$. Explicitly, if $\delta$ is a square root of $x^{2}-4$ in $\mathbb{C}$, then the eigenvalues are given by

$$
\begin{aligned}
\lambda & =\frac{x^{2}-2+x \delta}{2}, \\
\lambda^{-1} & =\frac{x^{2}-2-x \delta}{2} .
\end{aligned}
$$


The matrix $M$ is

- elliptic, if tr $M \in[-2,2) \Longleftrightarrow x \in(-2,2)$;

- parabolic, if $\operatorname{tr} M=2 \Longleftrightarrow x= \pm 2$;

- loxodromic, if tr $M \notin[-2,2] \Longleftrightarrow x \notin[-2,2]$.

CAsE $1 . M$ is elliptic, that is $x \in(-2,2)$. Let $\theta \in(0, \pi)$ such that

$$
\theta=\arccos \left(\frac{x^{2}-2}{2}\right) \text {. }
$$

In this case, there exists an invertible matrix $P$ such that

$$
M=P \cdot\left(\begin{array}{cc}
e^{i \theta} & 0 \\
0 & e^{-i \theta}
\end{array}\right) \cdot P^{-1} .
$$

So, the sequences $\left(y_{n}\right),\left(z_{n}\right)$ are given by

$$
\left(\begin{array}{l}
y_{n} \\
z_{n}
\end{array}\right)=\left(\begin{array}{l}
\mathfrak{y}(x) \\
\mathfrak{z}(x)
\end{array}\right)+P \cdot\left(\begin{array}{cc}
e^{i n \theta} & 0 \\
0 & e^{i n \theta}
\end{array}\right) \cdot P^{-1} \cdot\left[\left(\begin{array}{l}
y_{0} \\
z_{0}
\end{array}\right)-\left(\begin{array}{l}
\mathfrak{y}(x) \\
\mathfrak{z}(x)
\end{array}\right)\right] .
$$

Hence, the sequences $\left(y_{n}\right)$ and $\left(z_{n}\right)$ are bounded.

CASe 2. $M$ is parabolic, that is $x \in\{-2,2\}$. When $x=2$, we get the exact formulae:

$$
\begin{aligned}
& y_{n}=n^{2}(q-r)+n r-(2 n-1)\left(y_{0}+z_{0}\right) ; \\
& z_{n}=n^{2}(r-q)-n q+2 n\left(y_{0}+z_{0}\right) .
\end{aligned}
$$

Similarly, when $x=-2$, we get the formulae:

$$
\begin{aligned}
& y_{n}=n^{2}(q+r)-n r+(2 n-1)\left(z_{0}-y_{0}\right) ; \\
& z_{n}=n^{2}(q+r)+n q+2 n\left(z_{0}-y_{0}\right) .
\end{aligned}
$$

CASE 3. $M$ is loxodromic, that is $x \notin[-2,2]$. In particular, $x^{2} \neq 4$ and $x$ and $\delta$ are both non-zero. It follows that $I_{2}-M$ is invertible. We have the following formula:

$$
\left(\begin{array}{l}
y_{n} \\
z_{n}
\end{array}\right)=\left(\begin{array}{l}
\mathfrak{y}(x) \\
\mathfrak{z}(x)
\end{array}\right)+M^{n}\left[\left(\begin{array}{l}
y_{0} \\
z_{0}
\end{array}\right)-\left(\begin{array}{l}
\mathfrak{y}(x) \\
\mathfrak{z}(x)
\end{array}\right)\right]
$$


Diagonalizing $M$, we obtain the following formula

$$
M=P \cdot\left(\begin{array}{cc}
\lambda & 0 \\
0 & \lambda^{-1}
\end{array}\right) \cdot P^{-1},
$$

with

$$
P=\left(\begin{array}{cc}
\frac{\lambda^{-1}+1}{x} & \frac{\lambda+1}{x} \\
-1 & -1
\end{array}\right)
$$

Hence, a straightforward calculation gives

$$
M^{n}=\frac{1}{\sqrt{x^{2}-4}}\left(\begin{array}{cc}
\frac{\lambda+1}{x}\left(\lambda^{-n}-\lambda^{n-1}\right) & \lambda^{-n}-\lambda^{n} \\
\lambda^{n}-\lambda^{-n} & \frac{\lambda+1}{x}\left(\lambda^{n}-\lambda^{-n-1}\right)
\end{array}\right) .
$$

Using the definition of $\lambda$, we can see that

$$
\Lambda=\frac{\lambda+1}{x}=\frac{x+\delta}{2}
$$

is a square root of $\lambda$. And similarly

$$
\Lambda^{-1}=\frac{\lambda^{-1}+1}{x}=\frac{x-\delta}{2}
$$

is a square root of $\lambda^{-1}$. Moreover we have

$$
\delta=\Lambda-\Lambda^{-1} .
$$

So we obtain the following closed formulae for $y_{n}$ and $z_{n}$ :

$$
\begin{aligned}
& y_{n}=\frac{1}{\Lambda-\Lambda^{-1}}(-\Lambda^{2 n}\left(\Lambda^{-1}\left(y_{0}-\mathfrak{y}\right)+\left(z_{0}-\mathfrak{z}\right)\right) \\
&\left.+\Lambda^{-2 n}\left(\Lambda\left(y_{0}-\mathfrak{y}\right)+\left(z_{0}-\mathfrak{z}\right)\right)\right)+\mathfrak{y} ; \\
& z_{n}=\frac{1}{\Lambda-\Lambda^{-1}}\left(\Lambda^{2 n+1}\left(\Lambda^{-1}\left(y_{0}-\mathfrak{y}\right)+\left(z_{0}-\mathfrak{z}\right)\right)\right. \\
&\left.\quad-\Lambda^{-2 n-1}\left(\Lambda\left(y_{0}-\mathfrak{y}\right)+\left(z_{0}-\mathfrak{z}\right)\right)\right)+\mathfrak{z} .
\end{aligned}
$$


This means we can express these formulae as

$$
\begin{aligned}
& y_{n}=A \Lambda^{2 n}+B \Lambda^{-2 n}+\mathfrak{y} ; \\
& z_{n}=-\left(A \Lambda^{2 n+1}+B \Lambda^{-2 n-1}\right)+\mathfrak{z},
\end{aligned}
$$

with

$$
\begin{aligned}
& A=\frac{-1}{\Lambda-\Lambda^{-1}}\left(\Lambda^{-1}\left(y_{0}-\mathfrak{y}\right)+\left(z_{0}-\mathfrak{z}\right)\right), \\
& B=\frac{1}{\Lambda-\Lambda^{-1}}\left(\Lambda\left(y_{0}-\mathfrak{y}\right)+\left(z_{0}-\mathfrak{z}\right)\right) .
\end{aligned}
$$

Hence, we see that both sequences have the same behavior, up to sign. We have

$$
|\Lambda|=1 \Longleftrightarrow|\lambda|=1 \Longleftrightarrow x \in[-2,2] .
$$

Hence, when $x \notin[-2,2]$ and $A, B$ are both non-zero, the sequences $\left|y_{n}\right|$ and $\left|z_{n}\right|$ grow exponentially in $n$ and $-n$. To determine when at least one of $A, B=0$, we have the following identity concerning the product $A B$ :

$$
\begin{aligned}
A B & =\frac{-1}{\delta^{2}}\left(\frac{x+\delta}{2}\left(y_{0}-\mathfrak{y}\right)+\left(z_{0}-\mathfrak{z}\right)\right)\left(\frac{x-\delta}{2}\left(y_{0}-\mathfrak{y}\right)+\left(z_{0}-\mathfrak{z}\right)\right) \\
& =\frac{1}{4-x^{2}}\left(\left(y_{0}-\mathfrak{y}\right)^{2}+\left(\frac{x+\delta}{2}+\frac{x-\delta}{2}\right)\left(y_{0}-\mathfrak{y}\right)\left(z_{0}-\mathfrak{z}\right)+\left(z_{0}-\mathfrak{z}\right)^{2}\right) \\
& =\frac{1}{4-x^{2}}\left(y_{0}^{2}+x y_{0} z_{0}+z_{0}^{2}-y_{0}(2 \mathfrak{y}+x \mathfrak{z})-z_{0}(2 \mathfrak{z}+x \mathfrak{y})+\mathfrak{y}^{2}+\mathfrak{z}^{2}+x \mathfrak{y} \mathfrak{z}\right) \\
& =\frac{1}{4-x^{2}}\left(y_{0}^{2}+x y_{0} z_{0}+z_{0}^{2}-q y_{0}-r z_{0}+\frac{q^{2}+r^{2}-x r q}{4-x^{2}}\right) \\
& =\frac{1}{4-x^{2}}\left(p x+s-x^{2}+\frac{q^{2}+r^{2}-x r q}{4-x^{2}}\right) .
\end{aligned}
$$

Remark 3.7. The vertex relationship is

$$
y_{n}^{2}+x y_{n} z_{n}+z_{n}^{2}-q y_{n}-r z_{n}+x^{2}-p x-s=0,
$$

so it is a quadric and its type depends on the sign of the determinant

$$
D=\operatorname{det}\left[\begin{array}{ll}
1 & \frac{x}{2} \\
\frac{x}{2} & 1
\end{array}\right]=1-\frac{x^{2}}{4} .
$$


For detecting when the conic is degenerate we need the determinant

$$
\begin{aligned}
\Delta & =\operatorname{det}\left[\begin{array}{ccc}
1 & \frac{x}{2} & -\frac{q}{2} \\
\frac{x}{2} & 1 & -\frac{r}{2} \\
-\frac{q}{2} & -\frac{r}{2} & \left(x^{2}-p x-s\right)
\end{array}\right] \\
& =\frac{-x^{4}+p x^{3}+(4+s) x^{2}+2(r q-2 p) x-\left(q^{2}+r^{2}+4 s\right)}{4} .
\end{aligned}
$$

Note that

$$
\Delta=-\frac{A B\left(x^{2}-4\right)^{2}}{4} .
$$

We infer that the conic is degenerate when $x$ is a solution of the equation $\mathrm{AB}=0$. Following Benedetto and Goldman's notation [3], we can rewrite $\Delta$ as

$$
\Delta=-\frac{\kappa_{a, b}(x) \kappa_{c, d}(x)}{4},
$$

where $(a, b, c, d)$ are such that

$$
\mathrm{GT}(a, b, c, d)=(p, q, r, s)
$$

and

$$
\kappa_{a, b}(x)=x^{2}-a b x+a^{2}+b^{2}-4 .
$$

Let $u, v \in \mathbb{C}$ and denote by $x_{u, v}^{ \pm}$the two solutions of the equation $\kappa_{u, v}(x)=0$. Using this notation the solutions of the equation $A B=0$ are $x_{a, b}^{ \pm}, x_{c, d}^{ \pm}$.

We note that $A B$ does not depend on $y_{0}$ and $z_{0}$. The coefficients $A$ and $B$ are both non-zero unless $x \in\left\{x_{a, b}^{ \pm}, x_{c, d}^{ \pm}\right\}$. Note also that, in the case

$$
p=q=r=0,
$$

we recover the formula of Tan, Wong, and Zhang, namely

$$
A B=\frac{\left(x^{2}-s\right)}{\left(x^{2}-4\right)} .
$$

We can now conclude the discussion with the following lemma. 
Lemma 3.8. Suppose that $X \in \Omega_{1}$ has neighboring regions $Y_{n}$ and $Z_{n}, n \in \mathbb{Z}$.

(1) If $x \in(-2,2)$, then $\left|y_{n}\right|$ and $\left|z_{n}\right|$ remain bounded.

(2) If $x= \pm 2$, then $\left|y_{n}\right|$ and $\left|z_{n}\right|$ grow at most quadratically.

(3) If $x \notin[-2,2]$ and $x$ is not a solution of $A B=0$, then $\left|y_{n}\right|$ and $\left|z_{n}\right|$ grow exponentially as $n \rightarrow+\infty$ and as $n \rightarrow-\infty$.

(4) If $x \notin[-2,2]$ and $x$ is a solution of $A B=0$, then

- either

$$
\begin{aligned}
& \lim _{n \rightarrow-\infty} y_{n}=\mathfrak{y}, \\
& \lim _{n \rightarrow-\infty} z_{n}=\mathfrak{z}
\end{aligned}
$$

and both $\left|y_{n}\right|$ and $\left|z_{n}\right|$ grows exponentially as $n \rightarrow+\infty$,

- or

$$
\begin{aligned}
& \lim _{n \rightarrow+\infty} y_{n}=\mathfrak{y}, \\
& \lim _{n \rightarrow+\infty} z_{n}=\mathfrak{z}
\end{aligned}
$$

and both $\left|y_{n}\right|$ and $\left|z_{n}\right|$ grows exponentially as $n \rightarrow-\infty$,

- or

$$
y_{n}=\mathfrak{y} \quad \text { and } \quad z_{n}=\mathfrak{z}
$$

for all $n \in \mathbb{Z}$.

Given $\mu=\operatorname{GT}(a, b, c, d)$, let

$$
\mathcal{S}_{\boldsymbol{\mu}}:=\left\{x_{i, j}^{ \pm}:\{i, j\} \subset\{a, b, c, d\}\right\} .
$$

Note that the case (4) of the previous Lemma can only happen for a Markoff map $\phi$ if one of the neighboring regions takes value in $\mathcal{S}_{\boldsymbol{\mu}}$. In particular, the subcases of (4) correspond to the cases $A \neq 0, B \neq 0$ and $A=B=0$.

We can now give the definition of the constant $L(\mu)$ used to define the BQ-conditions. First define $M(\mu)$ as

$$
M(\mu)=\max \left\{\left|\frac{2 p_{i}-x p_{j}}{4-x^{2}}\right|: x \in \mathcal{S}(\mu) \backslash\{ \pm 2\}, p_{i} \neq p_{j} \in\{p, q, r\}\right\} .
$$

So $M(\mu)$ is the maximum modulus of the coordinates of the center of the conic equation taken on the finite number of cases where the conic is degenerate. 
Definition 3.9. Let

$$
L(\boldsymbol{\mu})=\max \{2+\alpha, M(\boldsymbol{\mu})+1\},
$$

and, similarly, let

$$
K(\boldsymbol{\tau})=L(\mathrm{GT}(\boldsymbol{\tau}))
$$

Lemma 3.10. Suppose $\beta$ is an infinite ray in $\Sigma$ consisting of a sequence $\left(e_{n}\right)_{n \in \mathbb{N}}$ of edges of $\Sigma$ such that the arrow on each $e_{n}$ assigned by $\phi$ is directed towards $e_{n+1}$. Then the ray $\beta$ meets at least one region $X$ with $|\phi(X)| \leq 2+\alpha$ and an infinite number of regions with $|\phi(X)| \leq L(\boldsymbol{\mu})$. Moreover, if $\phi^{-1}\left(\mathcal{S}_{\boldsymbol{\mu}}\right)=\emptyset$, then $\beta$ meets an infinite number of regions with $|\phi(X)| \leq 2+\alpha$.

Proof. - First we show that for any $\epsilon>0$, there exists a region $X$ adjacent to $\beta$ such that $\phi(X)<2+\alpha+\epsilon$.

Let

$$
\eta=\frac{\epsilon}{2+\alpha}
$$

and let $\left(X_{i}\right),\left(Y_{i}\right)$, and $\left(Z_{i}\right)$ the sequences of neighboring regions along the infinite ray. The sequences $\left(\left|x_{i}\right|\right),\left(\left|y_{i}\right|\right)$, and $\left(\left|z_{i}\right|\right)$ are decreasing and bounded below. So for $n$ large enough, we have two consecutive edges with region $X$ as a common face, i.e.

$$
\operatorname{vect}_{\phi}\left(e_{n}\right)=\left(X, Y ; Z^{\prime} \longrightarrow Z\right)
$$

and

$$
\operatorname{vect}_{\phi}\left(e_{n+1}\right)=\left(X, Z ; Y \longrightarrow Y^{\prime}\right),
$$

and with $|z| \leq\left|z^{\prime}\right| \leq|z|+\eta$. We can furthermore assume that $|y|>2$ and $|z|>2$. So we have easily

$$
\begin{aligned}
& |x z|=\left|y+y^{\prime}-q\right| \leq 2|y|+|q| ; \\
& |x y|=\left|z+z^{\prime}-r\right| \leq 2\left|z^{\prime}\right|+|r| \leq 2|z|+|r|+2 \eta .
\end{aligned}
$$

Multiplying both inequalities, we get

$$
|x|^{2} \cdot|y z| \leq 4|y z|+2|y| \cdot|r|+2|z| \cdot|q|+|q r|+\eta(2|q|+4|y|),
$$


So

$$
\begin{aligned}
|x|^{2} & \leq 4+2 \frac{|r|}{|z|}+2 \frac{|q|}{|y|}+\frac{|q r|}{|y z|}+\eta\left(\frac{4}{|z|}+\frac{2|q|}{|y z|}\right), \\
& \leq(2+\alpha)^{2}+\eta(2+\alpha) .
\end{aligned}
$$

Hence, we have that, for all $\epsilon>0$, there exists a region $X$ adjacent to $\beta$ such that $|\phi(X)|<2+\alpha+\epsilon$.

- Next, suppose that for all neighboring regions of the infinite ray, the values are always greater than $(2+\alpha)$. By the above argument, for all $\epsilon>0$ there exists a region $X$ such that $\phi(X)<2+\alpha+\epsilon$. So either $\phi(X) \leq 2+\alpha$, or, for all $\epsilon$, there exists an edge $e_{n}$ in the ray such that

$$
\operatorname{vect}_{\phi}\left(e_{n}\right)=\left(Y, Z ; X \longrightarrow X^{\prime}\right)
$$

with

$$
|x|+\left|x^{\prime}\right| \leq 2(2+\alpha)+\epsilon .
$$

Now the edge relation gives

$$
|x|+\left|x^{\prime}\right| \geq\left|x+x^{\prime}\right| \geq|y z-p| \geq|y z|-|p| .
$$

On the other hand, we have

$$
\begin{aligned}
|y z|-|p| & >(2+\alpha)^{2}-|p| \\
& >2(2+\alpha)+2 \alpha-|p|+\alpha^{2} \\
& >2(2+\alpha)+\alpha^{2} .
\end{aligned}
$$

Hence we have

$$
|x|+\left|x^{\prime}\right|>2(2+\alpha)+\alpha^{2} .
$$

Hence, if $\alpha \neq 0$, and $\epsilon$ is small enough, this gives a contradiction.

To conclude, we need to settle the case $\alpha=0$, which is equivalent to the case $p=q=r=0$. In this case the triple $(-x,-y,-z)$ is a $s$-Markoff triple in the sense of Tan, Wong, and Zhang, and the map is a Markoff map in their sense. So Lemma 3.11 of [28] gives the argument in this case, that is, there exists a region with $|\phi(X)|<2$. 
- For the second part of the statement, assume that there is only a finite number of regions meeting the ray such that $|\phi(X)|<2+\alpha$. Then, as the ray is decreasing, it means that one of the sequences $\left(X_{i}\right),\left(Y_{j}\right)$ or $\left(Z_{k}\right)$ that meet the infinite ray contains only a finite number of elements. Without loss of generality we can assume that it is the sequence $\left(X_{i}\right)$. Hence there exists $n_{0}$ such that for all $n \geq n_{0}$, the regions $Y_{n}$ and $Z_{n}$ are the neighbors of $X_{n_{0}}$. But we know that $\left(\left|y_{j}\right|\right)$ and $\left(\left|z_{k}\right|\right)$ are decreasing and bounded below, and from Lemma 3.8, the only possibility is that $X_{n_{0}} \in \phi^{-1}\left(\mathcal{S}_{\boldsymbol{\mu}}\right)$.

In addition, in the case where $X_{n_{0}} \in \phi^{-1}\left(\mathcal{S}_{\mu}\right)$, the sequences $\left(y_{n}, z_{n}\right)$ converges to the center of the degenerate conic whose coordinates are strictly smaller than $L(\boldsymbol{\mu})$. Hence, there is an infinite number of $n$ such that $\left|y_{n}\right|$ and $\left|z_{n}\right|$ are smaller than $L(\boldsymbol{\mu})$.

Example 3.11. We give an explicit example of an infinite descending ray which intersects only a finite number of regions with values less than $2+\alpha$. When $\boldsymbol{\mu}=(0 ; 0 ; 1 ; 20)$, take a Markoff map $\phi$ such that around a vertex $v\left(X, Y_{0}, Z_{0}\right)$,

$$
\begin{gathered}
x=\phi(X)=-\sqrt{3(4-\sqrt{7})} \approx-2.016 \geq-2-\alpha, \\
y_{0}=\phi(Y), \quad z_{0}=\phi\left(Z_{0}\right) \in \mathbb{R}, \quad y_{0}, z_{0}>33,
\end{gathered}
$$

and $\left(x, y_{0}, z_{0}\right)$ satisfy the vertex equation (such $y_{0}$ and $z_{0}$ will always exist). Then the neighboring sequences $y_{n}$ and $z_{n}$ around $X$ (as $n \rightarrow \infty$ ) are both decreasing and converging towards a fixed point $(16+6 \sqrt{7},(8+3 \sqrt{7}) \sqrt{3(4-\sqrt{7})}) \approx$ $(32,32)$. So this gives an infinite descending ray with only one neighboring region with value below $2+\alpha$.

We are now able to prove Theorem 3.1.

Proof of Theorem 3.1. The proof follows the arguments of [28], using Lemmata 3.3, 3.5 and 3.10, with details below.

(1) Let $l=l(\boldsymbol{\mu})=\max \{m(\boldsymbol{\mu}), 2+\alpha\}$. Suppose $\Omega_{\phi}(2+\alpha)$ is empty, then Lemmas 3.3 and 3.10 tell us that there exists a sink. Then Lemma 3.5 states that around a sink, one of the region is such that $|\phi(X)|<m(\mu)$.

(2) Suppose the statement is false, then choose a minimal path on $\Sigma$ joining two different connected components. We will use induction on the number of edges of this path. 
Suppose the path has one edge $e$. Without loss of generality we can assume $e \in E_{3}(\Sigma)$ and suppose $\vec{e}=(X, Y ; Z \rightarrow W)$. Then we have

$$
(2+\alpha)^{2}<|x y| \leq|r|+|z|+|w| \leq 2(\alpha)+2(2+\alpha),
$$

so $\alpha^{2}<0$, which is absurd.

Suppose now that the path has more than one edge, then the two ends of the path are directed outwards and we derive a contradiction by a simple induction argument on the length of the path using Lemma 3.3, as discussed in detail in the proof of Theorem 1 (2) of Bowditch [6].

\section{Domain of discontinuity}

The aim of this section is to prove Theorem A from the Introduction. In particular, we will prove that the set $\left(\boldsymbol{\Phi}_{\mu}\right)_{Q}$ of maps satisfying the BQ-conditions (where the constant $K$ is taken to be $L(\boldsymbol{\mu})$, equivalently $K(\boldsymbol{\tau})$ given by Definition 3.9) is an open subset of $\boldsymbol{\Phi}_{\boldsymbol{\mu}}$ in Theorem 4.19, and that the mapping class group acts properly discontinuously on it in Theorem 4.20. To do so, we will use the notion of Fibonacci growth, already used by Bowditch [6] and Tan, Wong and Zhang [28].

4.1. Fibonacci functions. In this section we recall the definition, given in [6], of Fibonacci function $F_{e}$ associated to an edge $e \in E(\Sigma)$, and of upper or lower Fibonacci bound for a function $f: \Omega \rightarrow[0, \infty)$.

Suppose $\vec{e} \in \vec{E}(\Sigma)$, and set $\Omega^{0}$ to be $\{X, Y\}$, where $e=X \cap Y$. Let $\Sigma^{ \pm}$be the two disjoint subtrees obtained by removing the interior of $e$ (such that $\Sigma^{+}$is at the head of $\vec{e}$ ), and let $\Omega^{ \pm}$be the set of regions whose boundaries lie in $\Sigma^{ \pm}$. We also denote

$$
\Omega^{0 \pm}=\Omega^{0} \cup \Omega^{ \pm} \text {. }
$$

First, we recall the notion of distance. Given $\vec{e} \in \vec{E}(\Sigma)$ with underlying edge $e$, we describe the function

$$
d: \Omega^{0-}(\vec{e}) \longrightarrow \mathbb{N}
$$

For $X \in \Omega^{0-}(\vec{e})$ we define

$$
d(X)=d_{\vec{e}}(X)
$$

to be the number of edges in the shortest path joining the head of $\vec{e}$ to $X$ (see Figure 3). Given any $Z \in \Omega^{-}(\vec{e})$, there are precisely two regions $X, Y \in \Omega^{0-}(\vec{e})$ meeting $Z$ and satisfying $d(X)<d(Z)$ and $d(Y)<d(Z)$. Note that $X, Y, Z$ all meet in a vertex. 
Now we can define the Fibonacci function

$$
F_{e}: \Omega \longrightarrow \mathbf{N}
$$

with respect to an edge $e$ as follows. We orient $e$ arbitrarily as $\vec{e}$, and use $-\vec{e}$ to denote the opposite orientation on $e$, and define

$$
F_{\vec{e}}: \Omega^{0-}(\vec{e}) \longrightarrow \mathbf{N}
$$

by

$$
F_{\vec{e}}(Z)= \begin{cases}1 & \text { if } Z \in \Omega^{0}(e), \\ F_{\vec{e}}(X)+F_{\vec{e}}(Y) & \text { if } Z \in \Omega^{-}(\vec{e}),\end{cases}
$$

and $X, Y \in \Omega^{0-}(\vec{e})$ are the two regions described above: $X \cap Y \cap Z \neq \varnothing$ and $d(X)<d(Z), d(Y)<d(Z)$. Now we define $F_{e}$ by

$$
F_{e}(X)= \begin{cases}F_{\vec{e}}(X) & \text { if } X \in \Omega^{0-}(\vec{e}), \\ F_{-\vec{e}}(X) & \text { if } X \in \Omega^{+}(\vec{e}) .\end{cases}
$$

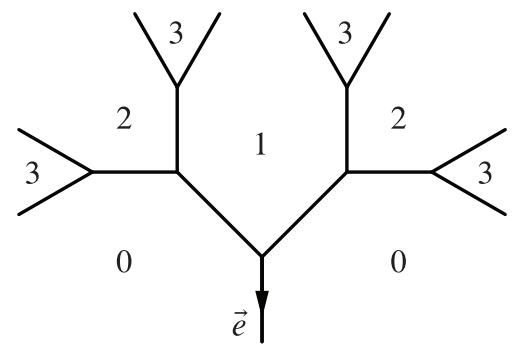

Figure 3. Distance function.

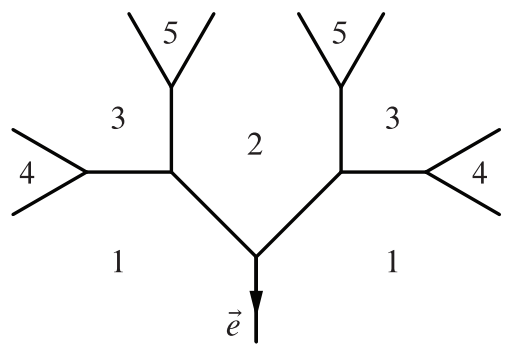

Figure 4. Fibonacci function $F_{\vec{e}}$.

The functions $F_{e}$ do not depend on the initial choice of the orientation $\vec{e}$ and provide a means for measuring the growth rates of functions defined on subsets of $\Omega$. The following lemma can be easily proved by induction. Its corollary shows that the concept of upper and lower Fibonacci bound is independent of the edge $e$ used. 
Lemma 4.1 (Lemma 2.1.1 in [6]). Suppose

$$
f: \Omega^{0-}(\vec{e}) \longrightarrow[0, \infty),
$$

where $\Omega^{0}(e)=\left\{X_{1}, X_{2}\right\}$.

(i) If $f$ satisfies $f(Z) \leq f(X)+f(Y)+c$ for some fixed constantc and arbitrary $X, Y, Z \in \Omega^{0-}(\vec{e})$ meeting at a vertex and satisfying $d(X)<d(Z)$ and $d(Y)<d(Z)$, then

$$
f(X) \leq(M+c) F_{e}(X)-c
$$

for all $X \in \Omega^{0-}(\vec{e})$, where $M=\max \left\{f\left(X_{1}\right), f\left(X_{2}\right)\right\}$.

(ii) If $f$ satisfies $f(Z) \geq f(X)+f(Y)-c$ for some fixed constant $c$, where $0<c<m=\min \left\{f\left(X_{1}\right), f\left(X_{2}\right)\right\}$ and arbitrary $X, Y, Z$ as in part (i), then

$$
f(X) \geq(m-c) F_{e}(X)+c
$$

for all $X \in \Omega^{0-}(\vec{e})$.

Corollary 4.2 (Corollary 2.1.2 in [6]). Suppose $f: \Omega \rightarrow[0, \infty)$ satisfies an inequality of the form $f(Z) \leq f(X)+f(Y)+c$ for some fixed constant $c$, whenever $X, Y, Z \in \Omega$ meet at a vertex. Then for any given edge $e \in E(\Sigma)$, there is a constant $K>0$, such that

$$
f(X) \leq K F_{e}(X)
$$

for all $X \in \Omega$.

Now we can define what it means for a function $f: \Omega \rightarrow[0, \infty)$ to have an upper or lower Fibonacci bound.

Definition 4.3. Suppose $f: \Omega \rightarrow[0, \infty)$, and $\Omega^{\prime} \subseteq \Omega$. We say that:

- $f$ has an upper Fibonacci bound on $\Omega^{\prime}$ if there is some constant $\kappa>0$ such that $f(X) \leq \kappa F_{e}(X)$ for all $X \in \Omega^{\prime}$;

- $f$ has a lower Fibonacci bound on $\Omega^{\prime}$ if there is some constant $\kappa>0$ such that $f(X) \geq \kappa F_{e}(X)$ for all but finitely many $X \in \Omega^{\prime}$;

- $f$ has Fibonacci growth on $\Omega^{\prime}$ if it has both upper and lower Fibonacci bounds on $\Omega^{\prime}$;

- $f$ has Fibonacci growth if $f$ has Fibonacci growth on all of $\Omega$. 
The following lemma tells us that, given an arbitrary $\boldsymbol{\mu}$-Markoff map $\phi$, the function $\log ^{+}|\phi|:=\max \{\log |\phi|, 0\}$ always has an upper Fibonacci bound on $\Omega$. Hence we will only need to consider criteria for it to have a lower Fibonacci bound on certain branches of the binary tree $\Sigma$ in order to determine if it has Fibonacci growth.

Lemma 4.4. If $\phi \in \boldsymbol{\Phi}_{\boldsymbol{\mu}}$, then $\log ^{+}|\phi|$ has an upper Fibonacci bound on $\Omega$.

Proof. We will follow the arguments of [28]. By Corollary 4.2 we only need to show that for an arbitrary $\boldsymbol{\mu}$-Markoff map $(x, y, z)$ we have

$$
\begin{aligned}
\log ^{+}|z| \leq & \log ^{+}|x|+\log ^{+}|y|+\log 10 \\
& +\log ^{+}|p|+\log ^{+}|q|+\log ^{+}|r|+\log ^{+}|s| .
\end{aligned}
$$

If $|z| \leq 2|x|$ or $|z| \leq 2|y|$, then (12) holds already. So we suppose $|z| \geq 2|x|$ and $|z| \geq 2|y|$. Then, since $p x+q y+r z+s-x y z=x^{2}+y^{2}+z^{2}$, we have

$$
\begin{aligned}
|p x|+|q y|+|r z|+|s|+|x y z| & \geq|z|^{2}-|x|^{2}-|y|^{2} \\
& =|z|^{2} / 2+\left(|z|^{2} / 4-|x|^{2}\right)+\left(|z|^{2} / 4-|y|^{2}\right) \\
& \geq|z|^{2} / 2 .
\end{aligned}
$$

Hence $|z|^{2} \leq 10 \max \{|p x|,|q y|,|r z|,|s|,|x y z|\}$, that is, according to the value of $\max \{|p x|,|q y|,|r z|,|s|,|x y z|\}$, we have, respectively,

(1) $|z|^{2} \leq 10|p x|$;

(2) $|z|^{2} \leq 10|q y|$;

(3) $|z|^{2} \leq 10|r z|$;

(4) $|z|^{2} \leq 10|s|$; or

(5) $|z|^{2} \leq 10|x y z|$.

Thus, since we may assume $|z| \geq 1$, we have, respectively,

(1) $|z| \leq|z|^{2} \leq 10|p x|$;

(2) $|z| \leq|z|^{2} \leq 10|q y|$

(3) $|z| \leq 10|r|$;

(4) $|z| \leq|z|^{2} \leq 10|s|$; or

(5) $|z| \leq 10|x y|$.

From this, Equation (12) follows easily. 
The lower Fibonacci bounds are more interesting since, as the following proposition shows, they give the convergence of certain series, as the following proposition shows, see [6].

Proposition 4.5 (Proposition 2.1.4 in [6]). If $f: \Omega \rightarrow[0, \infty)$ has a lower Fibonacci bound, then $\sum_{X \in \Omega} f(X)^{-s}$ converges for all $s>2$ (after excluding a finite subset of $\Omega$ on which $f$ takes the value 0 ).

The following lemma and corollary hold with proofs similar to the ones given in [6], giving a criterion for $\log ^{+}|\phi|$ to have a lower Fibonacci bound on certain branches of $\Sigma$.

Lemma 4.6. (i) Suppose $\vec{e} \in \vec{E}(\Sigma)$ is such that we have $\operatorname{vect}_{\phi}(e)=\vec{e}$ and that $\Omega^{0}(e) \cap \Omega_{\phi}(2+\alpha)=\emptyset$. Then

$$
\Omega^{0-}(\vec{e}) \cap \Omega_{\phi}(2+\alpha)=\emptyset
$$

and the arrow on each edge of $\Sigma^{-}$is directed towards $e$.

(ii) Furthermore,

$$
\log |\phi(X)| \geq(m-\log 2) F_{e}(X)
$$

for all $X \in \Omega^{0-}(\vec{e})$, where $m=\min \left\{\log |\phi(X)|: X \in \Omega^{0}(e)\right\}>\log (2+\alpha)$.

Corollary 4.7. If $\Omega_{\phi}(2+\alpha)=\emptyset$, then there exists a unique sink, and $\log ^{+}|\phi|$ has a lower Fibonacci bound.

Proof of Lemma 4.6. We will follow Bowditch's discussion [6]. Suppose that $\vec{e} \in \vec{E}(\Sigma)$ satisfies $\operatorname{vect}_{\Phi}(e)=\vec{e}$. Since $\Omega_{\phi}(2+\alpha)$ is connected, we must have either $\Omega_{\phi}(2+\alpha) \subset \Omega^{+}(\vec{e})$ or $\Omega_{\phi}(2+\alpha) \subset \Omega^{-}(\vec{e})$ (possibly $\Omega_{\phi}(2+\alpha)=\emptyset$ ). If $\Omega_{\phi}(2+\alpha) \subset \Omega^{-}(\vec{e})$ and $\Omega_{\phi}(2+\alpha) \neq \emptyset$, then $\alpha(e)=-\vec{e}$ (using an argument similar to the proof of Theorem 3.1 (ii)). This proves the first part of (i). For proving that arrows are directed towards $e$ one should use Lemma 3.3.

For the second part of the lemma, let $X, Y, Z \in \Omega^{0-}(\vec{e})$ meeting at a vertex and satisfying $d(X)<d(Z)$ and $d(Y)<d(Z)$, as in Lemma 4.1. Without loss of generality suppose $e \in E_{3}(\Sigma)$. By (i) we know that the arrow on $X \cap Y$ points away from $Z$. We know also that $|r| \leq 2 \alpha \leq|z| \alpha$. So we have that

$$
|x y| \leq 2|z|+|r| \leq(2+\alpha)|z| .
$$

Thus

$$
\log ^{+}|\phi(Z)| \geq \log ^{+}|\phi(X)|+\log ^{+}|\phi(Y)|-\log (2+\alpha) .
$$

We can now apply Lemma 4.1 (ii). 
Proof of Corollary 4.7. Again, following Bowditch's ideas, we recall that the existence comes from Lemma 3.10, the uniqueness from Lemma 3.3, while the upper Fibonacci bound comes from Lemma 4.4 and the lower one from Lemma 4.6.

Recall the definition of $\left(\boldsymbol{\Phi}_{\boldsymbol{\mu}}\right)_{Q}$ given in Section 2.4. We are ready to complete the aim of this subsection which is to prove the following result.

Theorem 4.8. Suppose $\phi \in\left(\boldsymbol{\Phi}_{\mu}\right)_{Q}$, then $\log ^{+}|\phi|$ has Fibonacci growth.

In order to prove it, we expand the discussion of Lemma 4.6 and we consider the case where $\Omega^{-}(\vec{e}) \cap \Omega_{\phi}(2)=\emptyset$ and exactly one of the two regions in $\Omega^{0}(e)$ has norm no greater than $2+\alpha$.

Lemma 4.9. Suppose that $\vec{e} \in \vec{E}(\Sigma)$ satisfies both the condition $\operatorname{vect}_{\phi}(e)=\vec{e}$ and the condition $\Omega^{0-}(\vec{e}) \cap \Omega_{\phi}(2+\alpha)=\left\{X_{0}\right\}$, where $X_{0} \in \Omega^{0}(\vec{e})$ with $x_{0} \notin[-2,2]$. Then $\log ^{+}|\phi|$ has a Fibonacci bound on $\Omega^{0-}(\vec{e})$.

Proof. Our proof slightly modifies Bowditch's proof of Lemma 3.8 in [6]. Let $\left(\vec{e}_{n}, \vec{f}_{n}\right)_{n=0}^{\infty}$ be the sequence of directed edges lying in the boundary of $X_{0}$ and in $\Omega^{0-}(\vec{e})$ so that $\vec{e}_{0}=\vec{e}$ and $\vec{e}_{n}$ is directed away from $\vec{f}_{n+1}$ and towards $\vec{f}_{n}$, and $\vec{f}_{n}$ is directed away from $\vec{e}_{n}$ and towards $\vec{e}_{n-1}$. For $n \geq 1$, let $v_{n}$ be the vertex incident on both $e_{n}$ and $f_{n}$ and $u_{n}$ be the vertex incident on both $e_{n}$ and $f_{n+1}$; let aso $\vec{\varepsilon}_{n}$ be the third edge (distinct from $e_{n}$ and $f_{n}$ ) incident on $v_{n}$ and directed towards $v_{n}$, and, similarly, let $\vec{\xi}_{n}$ be the third edge (distinct from $e_{n}$ and $f_{n+1}$ ) incident on $u_{n}$ and directed towards $u_{n}$. For $n \geq 0$, let $Y_{n}$ and $Z_{n}$ be the regions such that $Y_{n} \cap X_{0}=e_{n}$ and $Z_{n} \cap X_{0}=f_{n}$. (See Figure 5 for clarity.)

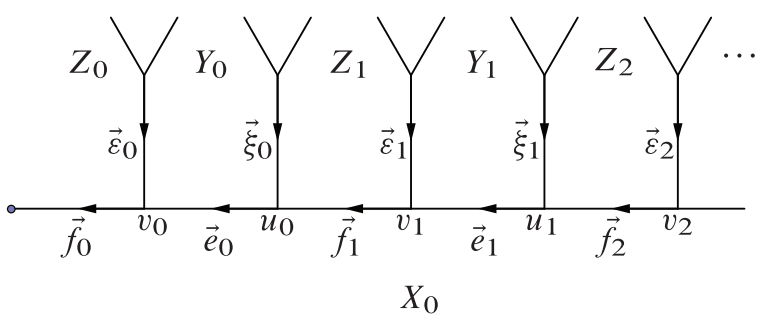

Figure 5. Neighbors around a region $X_{0}$.

Thus $\Omega^{0-}(\vec{e})=\left\{X_{0}\right\} \cup \bigcup_{n=1}^{\infty}\left(\Omega^{0-}\left(\vec{\varepsilon}_{n}\right) \cup \Omega^{0-}\left(\vec{\xi}_{n}\right)\right)$. We recall that, in this context, Bowditch noticed that, using Lemma 4.1(ii), a map $f: \Omega^{0-}(\vec{e}) \rightarrow[0, \infty)$ has a lower Fibonacci bound on $\Omega^{0-}(\vec{e})$ if and only if there is some constant $k>0$ such that for all $n \geq 1$ and for all $X \in \Omega^{0-}\left(\vec{\varepsilon}_{n}\right)$ we have $f(X) \geq k n F_{\vec{\varepsilon}_{n}}(X)$ and, similarly, for all $X \in \Omega^{0-}\left(\vec{\xi}_{n}\right)$ we have $f(X) \geq k n F_{\vec{\xi}_{n}}(X)$. 
By Lemma 3.8, $\left|y_{n}\right|$ and $\left|z_{n}\right|$ grow exponentially as $n \rightarrow \infty$, and so $\log \left|y_{n}\right| \geq c n$ and $\log \left|z_{n}\right| \geq c^{\prime} n$ for some constants $c, c^{\prime}>0$. Hence we have, for all $n \geq 1$, that $\log ^{+}|\phi(X)| \geq c n F_{\vec{\varepsilon}_{n}}(X)$, for all $X \in \Omega^{0-}\left(\vec{\varepsilon}_{n}\right)$, and that $\log ^{+}|\phi(X)| \geq c n F_{\vec{\xi}_{n}}(X)$, for all $X \in \Omega^{0-}\left(\vec{\xi}_{n}\right)$. Thus, using the characterisation above, it follows that $\log ^{+}|\phi|$ has a lower Fibonacci bound on $\Omega^{0-}(\vec{e})$.

Since the upper Fibonacci bound was proved in Lemma 4.4, the proof is done.

We can now prove Theorem 4.8. We first recall from [6] that the circular set $C(T)$ of a finite subtree $T \subset \Sigma$ consists of the set of directed edges $\vec{e}$ such that the intersection $\vec{e} \cap T$ is the head of $\vec{e}$.

Proof. The proof of Theorem 4.8 is the same as that of Theorem 2 in [6]. We sketch it as follows. By Lemma 4.4, we only need to show that $\log ^{+}|\phi|$ has a lower Fibonacci bound on $\Omega$. If $\Omega_{\phi}(2+\alpha)$ has at most one element, the conclusion follows easily by Corollary 4.7 and Lemma 4.9. Hence we can suppose $\Omega_{\phi}(2+\alpha)$ has at least two elements.

Recall that $\Omega_{\phi}(2+\alpha) \subseteq \Omega$ is finite and $\Omega_{\phi}(k)$ is connected for any $k \geq 2+\alpha$, see Theorem 3.1. Let $T$ be the (finite) subtree of $\Sigma$ spanned by the set of edges $e$ such that $\Omega^{0}(e) \subseteq \Omega_{\phi}(2+\alpha)$. Let $C=C(T)$ be the circular set of directed edges given by $T$. Note that $\Omega_{\phi}(2+\alpha)=\bigcup_{\vec{e} \in C} \Omega^{0-}(\vec{e})$. Hence it suffices to show that $\log ^{+}|\phi|$ has a lower Fibonacci bound on $\Omega^{0-}(\vec{e})$ for every $\vec{e} \in C$. Then the conclusion of Theorem 4.8 follows by the following lemma, Lemma 4.6 and Lemma 4.9.

Lemma 4.10. For each $\vec{e} \in C$, we have

$$
\begin{gathered}
\vec{e}=\operatorname{vect}_{\phi}(e), \\
\Omega^{-}(\vec{e}) \cap \Omega_{\phi}(2+\alpha)=\emptyset
\end{gathered}
$$

and $\Omega^{0}(e) \cap \Omega_{\phi}(2+\alpha)$ has at most one element.

Proof. Let $\vec{e}=(X, Y ; Z \rightarrow W) \in C(T)$. If one of $X$ and $Y$, say $X$, is in $\Omega_{\phi}(2+\alpha)$, then $Y, Z \notin \Omega_{\phi}(2+\alpha)$ and $W \in \Omega_{\phi}(2+\alpha)$ by the definition of $T$. Hence in this case $\vec{e}=\operatorname{vect}_{\phi}(e), \Omega^{-}(\vec{e}) \cap \Omega_{\phi}(2+\alpha)=\emptyset$ and $\Omega^{0}(e) \cap \Omega_{\phi}(2+\alpha)$ has one element, $X$.

Now suppose neither $X$ nor $Y$ is in $\Omega_{\phi}(2+\alpha)$, then $W \in \Omega_{\phi}(2+\alpha)$ and $Z \notin \Omega_{\phi}(2+\alpha)$ since $\Omega_{\phi}(2+\alpha)$ is connected. Thus in this case $\vec{e}=\operatorname{vect}_{\phi}(e)$, $\Omega^{0-}(\vec{e}) \cap \Omega_{\phi}(2+\alpha)=\emptyset$. This proves the lemma, completing the proof of the theorem. 
With Theorem 4.8 established, the following result becomes an easy corollary of Proposition 4.5.

Corollary 4.11. If $\phi \in\left(\boldsymbol{\Phi}_{\boldsymbol{\mu}}\right)_{Q}$, then for any $t>0$, the series $\sum_{X \in \Omega}|\phi(X)|^{t}$ converges absolutely.

4.2. Openness and properly discontinuous action. In this section we will prove Theorem A, using the definition of an attracting tree $T(t)$ for $t \geq 0$. In particular, first, we will define the attracting subtree $T(0)$, then we will use the existence of a certain map $H_{\mu}$, proved in Lemma 4.15, to define the attracting subtree $T(t)$.

4.2.1. Attracting tree $\boldsymbol{T}(\mathbf{0})$. From Lemma 3.8, we see that, when $x=\phi(X) \notin$ $[-2,2] \cup \mathcal{S}_{\boldsymbol{\mu}}$, the sequences $\left|y_{n}\right|$ and $\left|z_{n}\right|$ are monotonic for sufficiently large and sufficiently small $n$. (The set $\mathcal{S}_{\boldsymbol{\mu}}$ was defined at page 757.) From this observation, the following result follows easily.

Lemma 4.12. If $X \in \Omega$ and $\phi(X) \notin[-2,2] \cup \mathcal{S}_{\boldsymbol{\mu}}$, then there is a non-empty finite subarc $J(X) \subset \partial X$ with the property that, if $e$ is any edge in $\partial X$ not lying in $J(X)$, then the arrow on e points towards $J(X)$. Moreover, we can assume that $Y \cap X \subset J(X)$ for all $Y \in \Omega_{\phi}(2+\alpha)$.

When $x=\phi(X) \in[-2,2] \cup \mathcal{S}_{\mu}$, we shall set $J(X)=\partial X$.

Now, given $\phi \in \boldsymbol{\Phi}_{\boldsymbol{\mu}}$ with $\Omega_{\phi}(2+\alpha) \neq \emptyset$ (where $\alpha$ was introduced in Notation 3.2), we define

$$
T(0):=\bigcup_{X \in \Omega_{\phi}(2+\alpha)} J(X) .
$$

If $\Omega_{\phi}(2+\alpha)=\emptyset$, then, applying Corollary 4.7, we define $T(0)$ as the unique sink.

Lemma 4.13. $T(0)$ is connected, and the arrow on each edge not in $T(0)$ points towards $T(0)$.

Proof. The proof is elementary from Lemma 3.3. The arrows on every edge on the circular boundary of a connected component $T^{\prime}$ of $T(0)$ points towards $T^{\prime}$. Hence if we suppose there exists an arc outside $T(0)$ with its two endpoints on the boundary of $T(0)$, there is a vertex $v$ with two edges that point away from $v$, and hence the vertex $v$ would belong to $\Omega_{\phi}(2+\alpha)$, which gives rise to a contradiction. The same applies if an arrow outside $T(0)$ does not points toward $T(0)$. 
We say that $T(0)$ is an attracting subtree.

Corollary 4.14. If $\phi \in\left(\boldsymbol{\Phi}_{\boldsymbol{\mu}}\right)_{Q}$, then there is a finite attracting subtree $T(0)$.

Proof. If $\Omega_{\phi}(2+\alpha) \neq \emptyset$, then the tree $T(0)$ given by Lemma 4.13 is finite. Indeed, as $\phi \in\left(\boldsymbol{\Phi}_{\mu}\right)_{Q}$, there cannot be an infinite descending sequence (or bounded sequence) of regions and hence $\phi^{-1}\left([-2,2] \cup \mathcal{S}_{\mu}\right)=\emptyset$. If $\Omega_{\phi}(2+\alpha)=\emptyset$, then the result is true by definition.

4.2.2. Function $\boldsymbol{H}_{\boldsymbol{\mu}}$. Recalling the definition of $\mathcal{S}_{\boldsymbol{\mu}}$ given at page 757 , we can state the following result.

Lemma 4.15. There exists a function

$$
H_{\mu}: \mathbb{C} \backslash\left([-2,2] \cup \mathcal{S}_{\boldsymbol{\mu}}\right) \longrightarrow \mathbb{R}_{>0}
$$

so that for any $\phi \in \boldsymbol{\Phi}_{\boldsymbol{\mu}}$ and $X \in \Omega$, if $\left(Y_{n}\right)$ and $\left(Z_{n}\right)$ are the bi-infinite sequence of regions meeting $X$, then there are integers $n_{1} \leq n_{2}$ such that

$$
\left|y_{n}\right| \leq H_{\mu}(x) \text { and }\left|z_{n}\right| \leq H_{\mu}(x) \Longleftrightarrow n_{1} \leq n \leq n_{2}
$$

and $\left|y_{n}\right|$ and $\left|z_{n}\right|$ are monotomically decreasing for $n \in\left(-\infty, n_{1}\right)$ and monotonically increasing for $n \in\left[n_{2}, \infty\right)$. Moreover, $H_{\mu}(x) \geq 2+\alpha$.

Proof. From the formulae for $y_{n}$ and $z_{n}$ found at the end of the discussion of Case 3 in Section 3.2, it is an easy exercise, since $\left|y_{n}\right|$ and $\left|z_{n}\right|$ are asymptotic to exponentials in $|n|$ as $|n| \rightarrow \infty$.

Remark 4.16. A formula for $H_{\boldsymbol{\mu}}(x)$ would be quite hard to write explicitly.

As there is no ambiguity, we will use $H(x)$ in place of $H_{\mu}(x)$ for the rest of the section.

4.2.3. Attracting tree $T(t)$. For $x \in[-2,2] \cup \mathcal{S}_{\mu}$, we set

$$
H_{\mu}(x)=H(x)=\infty .
$$

Now for $X \in \Omega$ with $x=\phi(X) \notin[-2,2] \cup \mathcal{S}_{\mu}$ and for $r \geq H(x)$, we set

$$
J_{r}(X)=\bigcup\left\{\left(X \cap Y_{n}\right) \cup\left(X \cap Z_{n}\right):\left|y_{n}\right| \leq r \text { and }\left|z_{n}\right| \leq r\right\} .
$$

$J_{r}(X)$ is a subarc of $\partial X$ with the property that, if $e$ is any edge on $\partial X \backslash J_{r}(X)$, then the arrow on $e$ points towards $J_{r}(X)$. 
We now define an attracting subtree. Let

$$
T(t):=\bigcup_{X \in \Omega_{\phi}(2+\alpha+t)} J_{H(x)+t}(X) .
$$

Lemma 4.17. For all $t \geq 0$, the subtree $T(t)$ is connected and attracting. Moreover, if $t \geq m(\mu)-2-\alpha$, then $T(t) \neq \varnothing$.

We can describe $T(t)$ directly in terms of its edges. Suppose $e=X \cap Y$ is an edge. Then

$$
\begin{aligned}
e \in T(t) \Longleftrightarrow \text { either }|x| & \leq 2+\alpha+t \text { and }|y| \leq H(x)+t \\
\text { or }|y| & \leq 2+\alpha+t \text { and }|x| \leq H(y)+t .
\end{aligned}
$$

We can now prove the following lemma.

Lemma 4.18. For all $t \geq 0, \phi \in\left(\Phi_{\boldsymbol{\mu}}\right)_{Q}$ if and only if $T(t)$ is finite.

Proof. Let $\phi \in\left(\boldsymbol{\Phi}_{\boldsymbol{\mu}}\right)_{Q}$, then for each $X \in \Omega_{\phi}(2+\alpha+t)$ and $t \geq 0$, the arc $J_{H(x)+t}(X)$ has a finite number of edges as $x \notin[-2,2] \cup \mathcal{S}_{\mu}$. On the other hand, the lower Fibonacci bound proves that $\Omega_{\phi}(2+\alpha+t)$ is finite, hence $T(t)$ is finite.

Reciprocally, suppose $T(t)$ is finite. Then it is clear that $\phi^{-1}\left([-2,2] \cup \mathcal{S}_{\mu}\right)=\emptyset$. Now, for all $X \in \Omega_{\phi}(2+\alpha+t)$, the arc $J_{H(x)+t}(X)$ contains at least one edge. Hence we infer that $\Omega_{\phi}(2+\alpha+t)$ is finite, so $\phi \in\left(\boldsymbol{\Phi}_{\boldsymbol{\mu}}\right)_{Q}$.

Theorem 4.19. The set $\left(\Phi_{\mu}\right)_{Q}$ of maps satisfying the BQ-conditions is an open MeG-invariant subset of $\boldsymbol{\Phi}_{\boldsymbol{\mu}}$.

Proof. The invariance is easy. In fact, the conditions defining $\left(\boldsymbol{\Phi}_{\mu}\right)_{Q}$ only depend on the set of lengths of the simple closed curves, and the mapping class group does not change it.

Now, let $\phi \in\left(\boldsymbol{\Phi}_{\boldsymbol{\mu}}\right)_{Q}$, and $t_{1}>t_{0}>m(\boldsymbol{\mu})-2-\alpha$. By Lemma 4.18, $T\left(t_{1}\right)$ is a finite subtree of $\Sigma$, so we may choose $t_{2}>t_{1}$ large enough so that $T\left(t_{2}\right)$ contains $T\left(t_{1}\right)$ in its interior, that is, it contains $T\left(t_{1}\right)$ together with all the edges of the circular set $C\left(T\left(t_{1}\right)\right)$. Note that $T\left(t_{2}\right)$ is also a finite subtree of $\Sigma$. For any $\phi^{\prime} \in \boldsymbol{\Phi}_{\boldsymbol{\mu}}$, we write $T^{\prime}(t)$ for $T_{\phi^{\prime}}(t)$.

CLAIm If $\phi^{\prime}$ is sufficiently close to $\phi$, then $T^{\prime}\left(t_{1}\right) \cap T\left(t_{2}\right) \subseteq T\left(t_{1}\right)$. 
To prove the claim, choose an edge $e \in T\left(t_{2}\right) \backslash T\left(t_{1}\right)$ with neighboring regions $X$ and $Y$, so $e=X \cap Y$. Since $e \in T\left(t_{2}\right)$, we may assume $|\phi(X)| \leq 2+t_{2}+\alpha$ and $|\phi(Y)| \leq H(x)+t_{2}$. Then, as $e \notin T\left(t_{1}\right)$, we have $\left(|\phi(X)|>2+t_{1}+\alpha\right.$ or $\left.|\phi(Y)|>H(x)+t_{1}\right)$ and $\left(|\phi(Y)|>2+t_{1}+\alpha\right.$ or $\left.|\phi(X)|>H(y)+t_{1}\right)$. Thus, if $\phi^{\prime}$ is sufficiently close to $\phi$, the same inequalities hold when we replace $\phi$ with $\phi^{\prime}$. Hence $e \notin T^{\prime}\left(t_{1}\right)$. This proves the claim, since there are only finitely many edges in $T\left(t_{2}\right) \backslash T\left(t_{1}\right)$.

We know that $T(m(\boldsymbol{\mu})-2-\alpha)$ is a non-empty subtree of $T\left(t_{2}\right)$ and $t_{1}>m(\mu)-2-\alpha$, it follows that, for $\phi^{\prime}$ sufficiently close to $\phi$, we have $T^{\prime}\left(t_{1}\right) \cap T\left(t_{2}\right) \supseteq T(m(\boldsymbol{\mu})-2-\alpha) \neq \emptyset$.

Since $T^{\prime}\left(t_{1}\right) \cap T\left(t_{2}\right) \subseteq T\left(t_{1}\right)$ and $T\left(t_{1}\right)$ is contained in the interior of $T\left(t_{2}\right)$, we know that $T\left(t_{2}\right)$ contains a connected component of $T^{\prime}\left(t_{1}\right)$. Since $T^{\prime}\left(t_{1}\right)$ is connected, we must have $T^{\prime}\left(t_{1}\right) \subseteq T\left(t_{2}\right)$. Therefore $T^{\prime}\left(t_{1}\right)$ is finite, and so we conclude $\phi^{\prime} \in\left(\boldsymbol{\Phi}_{\boldsymbol{\mu}}\right)_{Q}$.

Theorem 4.20. The mapping class group acts properly discontinuously on the $\operatorname{set}\left(\boldsymbol{\Phi}_{\mu}\right)_{Q}$.

Proof. The statement is equivalent to the fact that $\operatorname{PSL}(2, \mathbb{Z})$ acts properly discontinuously on that set, that is, for any compact subset $K$ of $\left(\boldsymbol{\Phi}_{\mu}\right)_{Q}$, the set

$$
\{H \in \operatorname{PSL}(2, \mathbb{Z}): H K \cap K \neq \emptyset\}
$$

is finite. Suppose not, then there exists a sequence of distinct $H_{i} \in \operatorname{PSL}(2, \mathbb{Z})$ and $\phi_{i} \in K$ such that $H_{i}\left(\phi_{i}\right) \in K$. Passing to a subsequence, by the compactness of $K$, we may assume that $\phi_{i} \rightarrow \phi \in K, H_{i} \rightarrow \infty$, and $H_{i}\left(\phi_{i}\right) \rightarrow \phi^{\prime} \in K$. (Note that in a discrete group, for example $\operatorname{PSL}(2, \mathbb{Z})$, an infinite sequence of distinct elements $H_{i}$ tends to $\infty$.)

Now, as in the proof of Theorem 4.19, we have the tree $T_{\phi}\left(t_{1}\right)$ of $\phi$ is finite for some $t_{1}>0$, and that $T_{\phi_{i}}\left(t_{1}\right)$ is contained in the finite tree $T_{\phi}\left(t_{2}\right)$, for some $t_{2}>t_{1}$ and for all $i$ sufficiently large. This implies that the same constant $\kappa$ can be used in the lower Fibonacci bound for all $\phi_{i}$ for $i$ sufficiently large, and hence $H_{i}\left(\phi_{i}\right) \rightarrow \infty$ as $i \rightarrow \infty$. (Note that, in order to make sense of $\phi \rightarrow \infty$, we use the identification of $\boldsymbol{\Phi}_{\mu}$ with $X_{\tau}$, where $\mu=\mathrm{GT}(\boldsymbol{\tau})$, and hence with the character variety $\mathcal{V}=\left\{(x, y, z) \in \mathbb{C}^{3}: x^{2}+y^{2}+z^{2}+x y z=p x+q y+r z+s\right\}$; see the discussion in the Introduction and in Remark 2.2.) This contradicts $H_{i}\left(\phi_{i}\right) \rightarrow \phi^{\prime} \in K$. 


\section{The real case}

In this section we focus on the subset $X_{\tau}^{\mathbb{R}} \subset X_{\tau}$ of real characters $(x, y, z) \in \mathbb{R}^{3}$ with boundary data $\tau=(a, b, c, d) \in \mathbb{R}^{4}$. We will call this space the real character variety. The points of this space correspond to the real points of the complex relative character variety. The representations corresponding to these characters are representations of $\pi_{1}(S)$ into one of the two real forms of $\operatorname{SL}(2, \mathbb{C})$, namely $\mathrm{SL}(2, \mathbb{R})$ and $\mathrm{SU}(2)$. In particular, we will prove Theorem B from the Introduction, see Theorem 5.3 and Corollary 5.4.

5.1. Topology of the real character variety. In [3], Benedetto and Goldman completely described the topology of the set $X_{\tau}^{\mathbb{R}}$, when $\tau \in \mathbb{R}^{4}$. There are six different cases depending on the number $n$ of boundary traces in $[-2,2]$, that can vary from 0 to 4 :

(1) a quadruply punctured sphere, if $n=0$ and $a b c d<0$;

(2) a disjoint union of a triply punctured torus and a disc, if $n=0$ and $a b c d>0$;

(3) a disjoint union of a triply punctured sphere and a disc, if $n=1$;

(4) a disjoint union of an annulus and two discs, if $n=2$;

(5) a disjoint union of four discs, if $n=3$;

(6) a disjoint union of four discs and a sphere, if $n=4$.

Representations in $\mathrm{SU}(2)$ are such that $n=4$ and correspond to the compact connected component of $X_{\boldsymbol{\tau}}^{\mathbb{R}}$. However, when the parameters $\boldsymbol{\tau}=(a, b, c, d) \in$ $[-2,2]$ satisfy certain inequalities (see Proposition 1.4 of [3]), the compact component consists of representations in $\operatorname{SL}(2, \mathbb{R})$. The action of the mapping class group on the $\mathrm{SU}(2)$-character variety is ergodic by the work of Goldman [11].

The non-compact components always correspond to representations into $\operatorname{SL}(2, \mathbb{R})$, and in this case the dynamics of the action is richer. When $n=0$ the representations send boundary curves to hyperbolic elements of $\operatorname{SL}(2, \mathbb{R})$. In this case, a classic result of Goldman [10] states that the components are indexed by the relative Euler class which, according to Milnor-Wood inequality, is an element of $\{-2,-1,0,1,2\}$. 
So, in the case $n=0$, we have the following identifications.

- The disc component when $a b c d>0$ corresponds to hyperbolic structures on $S$ with geodesic boundary or equivalently to representations with maximal relative Euler class 2 or -2 ; see [3].

- The component in the case $a b c d<0$ corresponds to representations with relative Euler class 1 or -1 . Indeed, using the additivity of the relative Euler class, when we cut $S$ into two pairs of pants, the relative Euler class of one of them is 0 and the other is \pm 1 . Since the product of the three boundary traces is positive in the first pair of pants and negative in the other, we have $a b c d<0$.

- The triply punctured torus component corresponds to representations with relative Euler class 0 . For the same reason, when cutting $S$ into two pairs of pants the two relative Euler class are +1 and -1 , and hence both products of boundary traces are negative.

In the next section we will prove that the action of the mapping class group on the components corresponding to non-maximal Euler class are never ergodic on the whole component, except for a compact subset of dimension 1 of parameters $\boldsymbol{\tau}=(a, b, c, d) \in \mathbb{R}^{4}$ corresponding to the equations

$$
p(a, b, c, d)=q(a, b, c, d)=r(a, b, c, d)=0
$$

and $s \in[4,20]$. This is rather surprising as one could have expected that when $(p, q, r)$ are small enough, the dynamics of the action would be very close to the dynamics on the one-holed torus, which is known to be ergodic. In fact, domains of discontinuity will appear as soon as these parameters are non-zero; see Theorem 5.3 and Corollary 5.4.

5.2. Construction of a Markoff-map with BQ-conditions. We will prove in this section that, if the parameters $p, q$ and $r$ are not all zero, then the set of characters in $X_{\tau}^{\mathbb{R}}$ satisfying the BQ-condition is non-empty.

Lemma 5.1. Let $\boldsymbol{\mu}=(p, q, r, s)$ and suppose $(p, q, r) \neq(0,0,0)$. For all $\varepsilon>0$ and $K>0$, there exists a real $\boldsymbol{\mu}$-Markoff triple $\left(x_{1}, x_{2}, x_{3}\right)$ such that one of the values is $-(2+\varepsilon)<x_{i}<-2$ and the other two satisfy $x_{j} x_{k}>0$ and $\left|x_{j}\right|,\left|x_{k}\right|>K$. 
Proof. As $(p, q, r) \neq(0,0,0)$, we may assume, without loss of generality, that $(q, r) \neq(0,0)$ with $q r \geq 0$ (so $q$ and $r$ have the same sign). In this case, we search a $\mu$-Markoff triple of the form $(-(2+\epsilon), y, y)$ with $\epsilon>0$ and $y$ is such that $y(q+r)<0$ (so $y$ and $q$ are of opposite sign).

Such a point satisfies the equation

$$
(2+\epsilon)^{2}+2 y^{2}-(2+\epsilon) y^{2}+p(2+\epsilon)-(q+r) y-s=0 .
$$

This equation is quadratic in $\epsilon$ so we can express $\epsilon$ as a function of $y$ :

$$
\epsilon^{ \pm}(y)=\frac{1}{2}\left(\left(y^{2}-p-4\right) \pm \sqrt{y^{4}-8 y^{2}-2 p y^{2}+4(q+r) y+\left(p^{2}+4 s\right)}\right) .
$$

When $y$ gets large and we choose the solution with the minus sign, we have the following Taylor series:

$$
\begin{aligned}
\epsilon(y) \underset{y \rightarrow \infty}{=} \frac{1}{2}\left(\left(y^{2}-p-4\right)-y^{2} \sqrt{1+\left(\frac{-8-2 p}{y^{2}}+4 \frac{q+r}{y^{3}}+\frac{p^{2}+4 s}{y^{4}}\right)}\right) \\
\underset{y \rightarrow \infty}{=} \frac{1}{2}\left(\left(y^{2}-p-4\right)-y^{2}\left(1+\frac{1}{2}\left(\frac{-8-2 p}{y^{2}}+4 \frac{q+r}{y^{3}}\right)+o\left(\frac{1}{y^{3}}\right)\right)\right) \\
\underset{y \rightarrow \infty}{=}-\frac{q+r}{y}+o\left(\frac{1}{y}\right) .
\end{aligned}
$$

So taking $y$ large enough (in particular larger than $K$ ), we have $0<\epsilon(y)<\varepsilon$ and $|y|>K$. Hence the triple $(-2-\epsilon(y), y, y)$ is a $\boldsymbol{\mu}$-Markoff triple satisfying the conditions.

Lemma 5.2. Let $\boldsymbol{\mu}=(p, q, r, s)$ and suppose $(p, q, r) \neq(0,0,0)$. Then there exists a $\boldsymbol{\mu}$-Markoff map $\phi \in \mathbf{\Phi}_{\boldsymbol{\mu}}$ such that $\left|\Omega_{\phi}(2+\alpha)\right|$ is finite.

Proof. As the three numbers $p, q$ and $r$ are not all zero, there are two of them that are of the same sign and not both zero. Without loss of generality, we can assume that $(q, r) \neq(0,0)$ and $q \leq 0$ and $r \leq 0$ (the positive case can be treated the same way). We know from the previous lemma that we can find a $\boldsymbol{\mu}$-Markoff triple $(-2-\epsilon(y), y, y)$, with $y>0$ large, and $\epsilon(y)$ is a function of $y$ whose Taylor series is $\epsilon(y)=-\frac{q+r}{y}+o\left(\frac{1}{y}\right)$. Let $\phi$ be the corresponding $\mu$-Markoff map. Let's call $X_{0}, Y_{0}$ and $Z_{0}$ the regions corresponding to this triple. The neighbors around $X_{0}$ are denoted by the two sequences $Y_{n}$ and $Z_{n}$.

The values of the neighbors of $X_{0}$ are of the form:

$$
\begin{aligned}
& y_{n+1}=q+(2+\epsilon) z_{n}-y_{n} ; \\
& z_{n+1}=r+(2+\epsilon) y_{n+1}-z_{n} .
\end{aligned}
$$


- First assume that $q$ and $r$ are both non-zero so that $-(q+r)>\max \{-q,-r\}$. Hence, we can choose $y_{0}=z_{0}=y$ large enough so that

$$
\epsilon=\epsilon(y)>\frac{\max \{-q,-r\}}{y}>0 .
$$

In this case, we can prove by recurrence that for all $n>0$ we have

$$
y_{n+1}>z_{n}>y_{n}>y .
$$

Indeed for $n=1$ we have

$$
\begin{aligned}
y_{1} & =q+(2+\epsilon) y-y \\
& =y+\epsilon y+q>y ; \\
z_{1} & =r+(2+\epsilon) y_{1}-y \\
& =y_{1}+\left(y_{1}-y\right)+r+y_{1} \epsilon>y_{1} ; \\
y_{2} & =q+(2+\epsilon) z_{1}-y_{1} \\
& >z_{1}+\left(z_{1}-y_{1}\right)+\epsilon z_{1}+q>z_{1} .
\end{aligned}
$$

The induction follows the same steps:

$$
\begin{aligned}
z_{n+1} & =r+(2+\epsilon) y_{n+1}-z_{n} \\
& =y_{n+1}+\left(y_{n+1}-z_{n}\right)+y_{n+1} \epsilon+r \\
& >y_{n+1}+y \epsilon+r \\
& >y_{n+1} \\
y_{n+2} & =q+(2+\epsilon) z_{n+1}-y_{n} \\
& >z_{n+1}+\left(z_{n+1}-y_{n+1}\right)+z_{n+1} \epsilon+q \\
& >z_{n+1} .
\end{aligned}
$$

A similar treatment can be applied to $n<0$, in which case we have

$$
z_{n-1}>y_{n}>z_{n}>y .
$$

This proves that all neighbors of $X_{0}$ have value greater than $y$. As we can choose $y$ to be greater than $2+\alpha$, and the set $\Omega_{\phi}(2+\alpha)$ is connected, it is clear that $X_{0}$ is the only region in $\Omega_{\phi}(2+\alpha)$. 
- Now assume one of $q$ or $r$ is zero, for example $r=0$. We can choose $y$ large enough so that $\epsilon=\epsilon(y)>-\frac{3}{4} \frac{q}{y}$ and $\epsilon<\frac{1}{4}$. In this case we can prove that for all $n \geq 2$ we have

$$
y_{n+1}>z_{n}>y_{n}-\frac{3}{4} q \text { and } y_{n} \geq y .
$$

Indeed for the first terms of the sequence, we have

$$
\begin{aligned}
y_{1} & =q+(2+\epsilon) y-y=y+(q+\epsilon y) \\
& >y+\frac{q}{4} ; \\
z_{1} & =(2+\epsilon) y_{1}-y \\
& >y_{1}+\left(y_{1}-y\right)+y_{1} \epsilon \\
& >y_{1}+\frac{q}{4}-\frac{3 q}{4}+\epsilon \frac{q}{4} \\
& >y_{1}-\frac{q}{2} ; \\
y_{2} & =q+(2+\epsilon) z_{1}-y_{1} \\
& >z_{1}+\left(z_{1}-y_{1}\right)+\epsilon(y) z_{1}>z_{1} ; \\
z_{2} & =(2+\epsilon) y_{2}-z_{1} \\
& =y_{2}+\left(y_{2}-z_{1}\right)+\epsilon y_{2}>y_{2}-\frac{3 q}{4} ; \\
y_{3} & =q+(2+\epsilon) z_{2}-y_{2} \\
& >z_{2}-\frac{q}{2} .
\end{aligned}
$$

The induction follows the same steps:

$$
\begin{aligned}
z_{n+1} & =(2+\epsilon) y_{n+1}-z_{n} \\
& =y_{n+1}+\left(y_{n+1}-z_{n}\right)+y_{n+1} \epsilon \\
& >y_{n+1}+y \epsilon \\
& >y_{n+1}-\frac{3 q}{4} ;
\end{aligned}
$$




$$
\begin{aligned}
y_{n+2} & =q+(2+\epsilon) z_{n+1}-y_{n} \\
& >z_{n+1}+\left(z_{n+1}-y_{n+1}\right)+z_{n+1} \epsilon+q \\
& >z_{n+1}-\frac{3 q}{4}-\frac{3 q}{4}+q \\
& >z_{n+1} .
\end{aligned}
$$

Similarly for $n \leq-2$, we have the same results. In conclusion in all cases, the set $\Omega_{\phi}(2+\alpha)$ is finite.

To conclude, it remains to show that the Markoff map $\phi$ constructed in the previous lemma satisfies the BQ-conditions. First, note that $\Omega_{\phi}(2)$ is empty, as we constructed an attracting subtree such that the value of all regions adjacent to this tree are greater than 2. Now, using the proof of Theorem 4.8, we see that, if $\Omega_{\phi}(2+\alpha)$ is finite and $\phi^{-1}\left([-2,2] \cup \mathcal{S}_{\boldsymbol{\mu}}\right)=\emptyset$, then $\log ^{+}|\phi|$ has Fibonacci growth, and hence $\Omega_{\phi}(L)$ is finite. In addition, since in the construction of $\phi$ we can choose any $y>0$ large enough as a starting point, and since $\mathcal{S}_{\boldsymbol{\mu}}$ is finite, we can see that we can choose $\phi$ such that $\phi^{-1}\left(\mathcal{S}_{\mu}\right)=\emptyset$.

We can now state the theorem.

Theorem 5.3. Let $\boldsymbol{\mu}=(p, q, r, s)$ and suppose $(p, q, r) \neq(0,0,0)$. Then the set $\left(\boldsymbol{\Phi}_{\boldsymbol{\mu}}\right)_{Q}^{\mathbb{R}}$ is non-empty.

We will use this in the next section.

5.3. Domain of discontinuity. The result of the previous section and the arguments discussed before for the (complex) character variety (adapted to this setting) implies the following result.

Corollary 5.4. Let $\boldsymbol{\tau}=(a, b, c, d) \in \mathbb{R}^{4}$. We have the following cases depending on $(a, b, c, d)$ for the dynamics of the action of the mapping class group on $X_{\tau}^{\mathbb{R}}$.

(1) if $|a|=|b|=|c|=|d|$ with abcd $\leq 0$ and $2<|a| \leq \sqrt{2(1+\sqrt{5})}$, then the action is ergodic on the whole real relative character variety $X_{\tau}^{\mathbb{R}}$.

(2) If $|a|=|b|=|c|=|d|$ with abcd $\leq 0$ and $|a|=0$ or $|a|=2$, then the action is ergodic on the invariant subset $K=X_{\tau}^{\mathbb{R}} \cap[-2,2]^{3}$ and is also ergodic on each of the four components of $X_{\tau}^{\mathbb{R}} \backslash K$.

(3) In all other cases, $\left(X_{\tau}^{\mathbb{R}}\right)_{Q}$ is a non-empty open domain of discontinuity for the action of the mapping class group on $X_{\tau}^{\mathbb{R}}$. 
Remark 5.5. A $\mathcal{M} \mathcal{L} \mathcal{G}$-invariant measure $\mu$ can be constructed using the natural symplectic structure on the relative character variety, defined by Goldman [11], and ergodicity can be stated with respect to this measure. However, note that for ergodicity results only the class of the measure is relevant, and the measure $\mu$ is in the usual Lebesgue class.

Proof. In the case $(p, q, r)=(0,0,0)$, the set of $(0,0,0, s)$-Markoff maps is identified with the set of $s$-Markoff maps in the sense of Tan-Wong-Zhang. These maps correspond to real characters of the one-holed torus. The action of the mapping class group on the set of real characters $\Phi_{s}^{\mathbb{R}}$ is completely described by Goldman [12], and can be stated as follows.

- If $s<0$, there are four contractible connected components in the character variety and the action is properly discontinuous on each one.

- If $s \in[0,4[$, there are four contractible connected components on which the action is properly discontinuous, and one compact component where the action is ergodic.

- If $s=4$, the set of real characters is connected but contains the invariant subset of characters of reducible $\mathrm{SU}(2)$-representations as a proper subset $K$, which corresponds to characters in $[-2,2]^{3}$, and the action is ergodic on $K$. The complementary set consists of four connected components, and the action is ergodic on each of these components.

- If $s \in(4,20]$, there is a unique connected component and the action of the mapping class group is ergodic.

- If $s>20$, there is a unique connected component. There is an open domain $\mathcal{D}$ which is a domain of discontinuity for the action, and the action is ergodic on the complement of $\mathcal{D}$.

When $(p, q, r)$ is non-zero, there is a non-empty open domain of discontinuity by Theorem 5.3. So the only case we need to settle is the case of $(p, q, r)=(0,0,0)$.

The case (2) corresponds exactly to the situation when $s=4$. Also we note that if $(p, q, r)=(0,0,0)$, the domain of discontinuity is non-empty as soon as $s \notin[4,20]$, and that when $s \in(4,20]$, the action is ergodic on the whole character variety.

From Goldman and Toledo [13], we have $(p, q, r)=(0,0,0)$ if and only if one of the following is satisfied:

(1) $|a|=|b|=|c|=|d|$ with $a b c d \leq 0$;

(2) three of $a, b, c, d$ are zero. 
In the first case $s=4-a^{2}-b^{2}-c^{2}-d^{2}-a b c d=4-4 a^{2}+a^{4}$. Solving the system $4<4-4 a^{2}+a^{4} \leq 20$ gives

$$
2<|a| \leq \sqrt{2(1+\sqrt{5})}
$$

Else, the equation $s=4$ implies that $|a|=0$ or $|a|=2$.

In the second case, without loss of generalities, we can assume that $b=c=$ $d=0$, which gives $s=4-a^{2} \leq 4$. Hence $s \notin(4,20]$ if and only if $|a| \neq 0$, which ends the proof.

\section{Concluding remarks}

This paper was mostly concerned with describing a domain of discontinuity for the action of $\mathcal{M C G}(S)$ on the (relative) character variety of the four-holed sphere $S$. We expect that it would be possible to implement this on a program to draw various slices of the domain of discontinuity, and we expect that, in general, these slices would have highly fractal boundary, as in the case of the character variety for the free group $F_{2}$ on two generators analysed by Series, Tan and Yamashita [26]. Restriction to the real case may also be of interest. It is not clear at this point if the domains of discontinuity for the real case would have relatively smooth boundary as in the case analysed by Goldman in [12] for $F_{2}$, or if they would exhibit fractal type boundary.

There are also other group actions on the character variety which are of interest. In particular, we can consider the action of $\operatorname{Out}\left(F_{3}\right)$ as studied by Minsky [22] (this is a bigger group action than what we consider). In this case, it is somewhat difficult to give easily (computer) verifiable conditions to describe the domain of discontinuity like the BQ-conditions, nonetheless, our methods may provide a starting point towards this end.

Finally we note that, as pointed out in the beginning of the Introduction, much of the original motivation for the paper came from McShane's identity and the proof provided by Bowditch. A new generalization to the identity from the point of view of Coxeter group actions on quartic varieties was given by $\mathrm{Hu}$, Tan and Zhang in [14], and we plan to pursue this direction in a future paper and explore possible generalizations of the McShane's identity in our context. 


\section{References}

[1] H, Akiyoshi, H, Miyachi, and M. Sakuma, Refinement of McShane's identity for quasifuchsian punctured torus groups. A W. Abikoff and A. Haas (eds.), In the tradition of Ahlfors and Bers. III. (University of Connecticut, 2001.) Contemporary Mathematics, 355. American Mathematical Society, Providence, R.I., 2004, 21-40. Zbl 1080.30038 MR 2145054

[2] H. Akiyoshi, H. Miyachi, and M. Sakuma (eds.), Variations of McShane's identity for punctured surface groups. In Y. N. Minsky, M. Sakuma, and C. Series (eds.), Spaces of Kleinian groups. (Cambridge 2003.) London Mathematical Society Lecture Note Series, 329. Cambridge University Press, Cambridge, 2006, 151-185. Zbl 1103.30027 MR 2258748

[3] R. L. Benedetto and W. M. Goldman, The topology of the relative character varieties of a quadruply-punctured sphere. Experiment. Math. 8 (1999), no. 1, 85-103. Zbl 0957.57003 MR 1685040

[4] B. H. Bowditch, A proof of McShane's identity via Markoff triples. Bull. London Math. Soc. 28 (1996), no. 1, 73-78. Zbl 0854.57009 MR 1356829

[5] B. H. Bowditch, A variation of McShane's identity for once-punctured torus bundles. Topology 36 (1997), no. 2, 325-334. Zbl 0872.57015 MR 1415591

[6] B. H. Bowditch, Markoff triples and quasi-Fuchsian groups. Proc. London Math. Soc. (3) 77 (1998), no. 3, 697-736. Zbl 0928.11030 MR 1643429

[7] S. Bratholdt and D. Cooper, On the topology of the character variety of a free group. Rend. Istit. Mat. Univ. Trieste 32 (2001), suppl. 1, 45-53. Dedicated to the memory of M. Reni. Zbl 1062.14505 MR 1889465

[8] S. Cantat and F. Loray, Dynamics on character varieties and Malgrange irreducibility of Painlevé VI equation. Ann. Inst. Fourier (Grenoble) 59 (2009), no. 7, 2927-2978. Zbl 1204.34123 MR 2649343

[9] R. Fricke and F. Klein, Vorlesungen über die Theorie der automorphen Funktionen. Teubner, Leipzig, 1897. Reprint, Bibliotheca Mathematica Teubneriana, Bände 3, 4. Johnson Reprint Corp., New York, and B. G. Teubner, Stuttgart, 1965. MR 0183872

[10] W. M. Goldman, Topological components of spaces of representations. Invent. Math. 93 (1988), no. 3, 557-607. Zbl 0655.57019 MR 0952283

[11] W. M. Goldman, Ergodic theory on moduli spaces. Ann. of Math. (2) 146 (1997), no. 3, 475-507. Zbl 0907.57009 MR 1491446

[12] W. M. Goldman, The modular group action on real SL(2)-characters of a one-holed torus. Geom. Topol. 7 (2003), 443-486. Zbl 1037.57001 MR 2026539

[13] W. M. Goldman and D. Toledo, Affine cubic surfaces and relative SL(2)-character varieties of compact surfaces. Preprint 2010. arXiv:1006.3838v2 [math.GT]

[14] H. Hu, S. P. Tan, and Y. Zhang, Polynomial automorphisms of $\mathbb{C}^{n}$ preserving the Markoff-Hurwitz equation. Preprint 2012. arXiv:1501.06955v2 [math.GT] 
[15] L. Keen and C. Series, The Riley slice of Schottky space. Proc. London Math. Soc. (3) 69 (1994), no. 1, 72-90. Zbl 0807.30031 MR 1272421

[16] F. Labourie and G. McShane, Cross ratios and identities for higher TeichmüllerThurston theory. Duke Math. J. 149 (2009), no. 2, 279-345. Zbl 1182.30075 MR 2541705

[17] D. Lee and M. Sakuma, A variation of McShane's identity for 2-bridge links. Geom. Topol. 17 (2013), no. 4, 2061-2101. Zbl 1311.57022 MR 3109863

[18] W. Magnus, Rings of Fricke characters and automorphism groups of free groups. Math. Z. 170 (1980), no. 1, 91-103. Zbl 0433.20033 MR 0558891

[19] G. McShane, A remarkable identity for lengths of curves. Ph.D. thesis, University of Warwick, Coventry, 1991.

[20] G. McShane, Simple geodesics and a series constant over Teichmüller space. Invent. Math. 132 (1998), no. 3, 607-632. Zbl 0916.30039 MR 1625712

[21] Y. N. Minsky, The classification of punctured-torus groups. Ann. of Math. (2) 149 (1999), no. 2, 559-626. Zbl 0939.30034 MR 1689341

[22] Y. N. Minsky, On dynamics of $\operatorname{Out}\left(F_{n}\right)$ on $\mathrm{PSL}_{2}(\mathbb{C})$ characters. Israel J. Math. 193 (2013), no. 1, 47-70. Zbl 1282.57023 MR 3038545

[23] M. Mirzakhani, Simple geodesics and Weil-Petersson volumes of moduli spaces of bordered Riemann surfaces. Invent. Math. 167 (2007), no. 1, 179-222. Zbl 1125.30039 MR 2264808

[24] F. Palesi, Ergodic actions of mapping class groups on moduli spaces of representations of non-orientable surfaces. Geom. Dedicata 151 (2011), 107-140. Zbl 1213.57005 MR 2780741

[25] M. Sakuma, Variations of McShane's identity for the Riley slice and 2-bridge links. Sūrikaisekikenkyūsho Kōkyūroku 1104 (1999), 103-108. Zbl 0951.57500 MR 1744474

[26] C. Series, S. P. Tan, and Y. Yamashita, The diagonal slice of Schottky space. Preprint 2014. arXiv:1409.6863 [math.GT]

[27] S. P. Tan, Y. L. Wong, and Y. Zhang, Generalizations of McShane's identity to hyperbolic cone-surfaces. J. Differential Geom. 72 (2006), no. 1, 73-112. Zbl 1097.53031 MR 2215456

[28] S. P. Tan, Y. L. Wong, and Y. Zhang, Generalized Markoff maps and McShane's identity. Adv. Math. 217 (2008), no. 2, 761-813. Zbl 1158.57023 MR 2370281

[29] S. P. Tan, Y. L. Wong, and Y. Zhang, McShane's identity for classical Schottky groups. Pacific J. Math. 237 (2008), no. 1, 183-200. Zbl 1165.30025 MR 2415214 
Received April 29, 2013

Sara Maloni, Department of Mathematics, Brown University, Box 1917, 151 Thayer Street, Providence, RI 02912, USA

home page: http://www.math.brown.edu/ maloni

email: sara_maloni@brown.edu

Frédéric Palesi, Institut de Mathématiques de Marseille, Aix Marseille Université, CNRS, Centrale Marseille, I2M, UMR 7373, 39 rue F. Joliot Curie, 13453 Marseille Cedex 13, France

home page: http://www.latp.univ-mrs.fr/ fpalesi

email: frederic.palesi@univ-amu.fr

Ser Peow Tan, Department of Mathematics, National University of Singapore, Block S17, 10 Lower Kent Ridge Road, Singapore 119076

home page: http://www.math.nus.edu.sg/ mattansp

email: mattansp@nus.edu.sg 